\title{
Synthesis of $N$-acyl sulfamates from fluorosulfonates and potassium trimethylsilyloxyl imidates
}

\section{Supporting information}

Shuning Zhang a,b, Huan Xiong a, Fengping ${ }^{\text {Lu }}{ }^{\text {a }}$, Fei Ma ${ }^{\text {a }}$, Yuang $\mathrm{Gu}^{\text {a }}$, Peixiang Ma ${ }^{\mathrm{a},{ }^{*}}$, Hongtao $\mathrm{Xu}^{\mathrm{a},{ }^{*}}$ and Guang Yang a,* China

a Shanghai Institute for Advanced Immunochemical Studies, ShanghaiTech University, 201210 Shanghai,

${ }^{b}$ School of Life Science and Technology, ShanghaiTech University, 201210 Shanghai, China

\section{Contents}

${ }^{1} \mathrm{H}$ and ${ }^{13} \mathrm{C}$ NMR spectra of new compouns. . $\mathrm{S} 2$ 


\section{${ }^{1} \mathrm{H}$ and ${ }^{13} \mathrm{C}$ NMR spectra of new compounds}

1-(4-methoxyphenyl)piperazine (1c1):

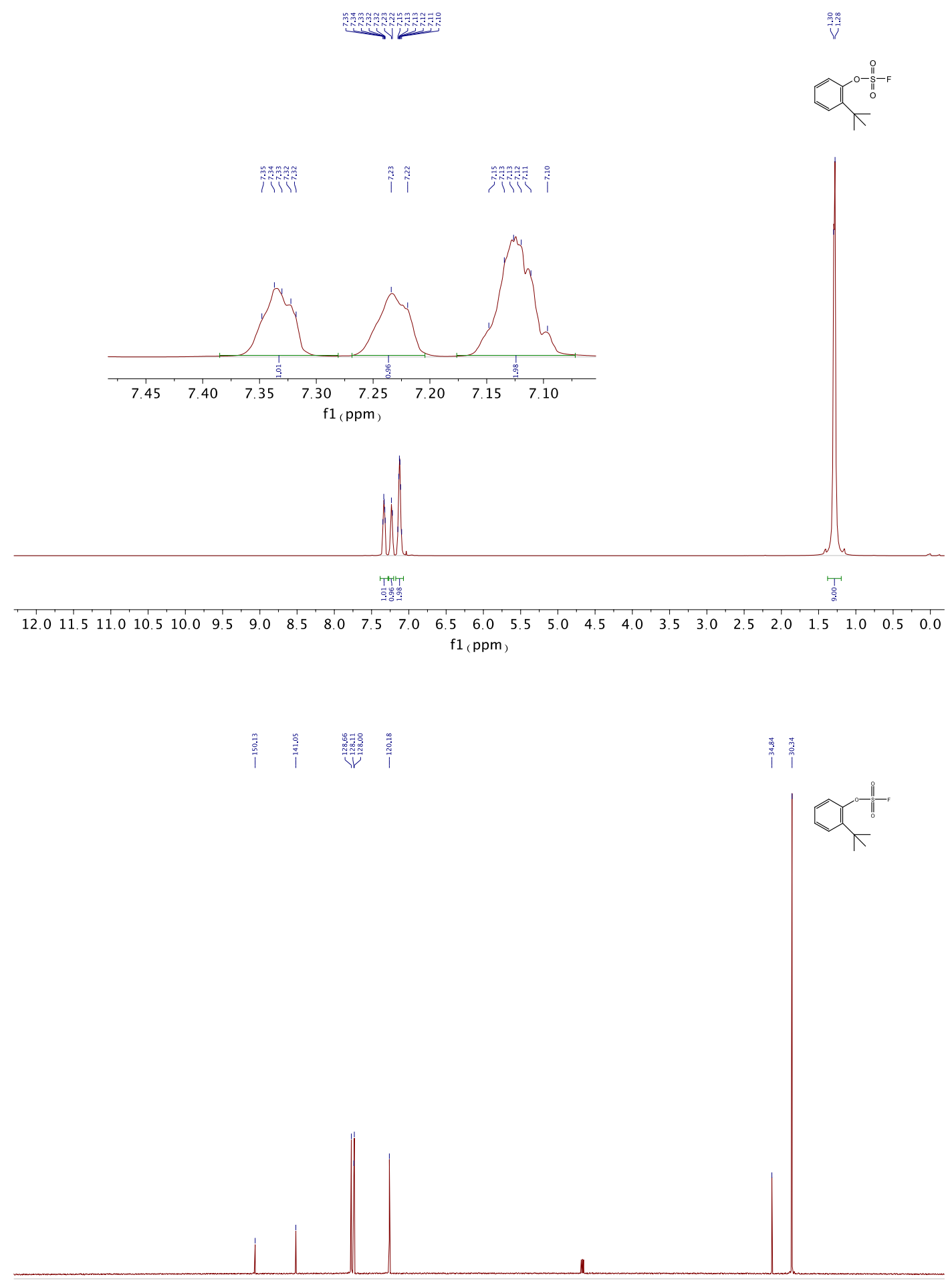

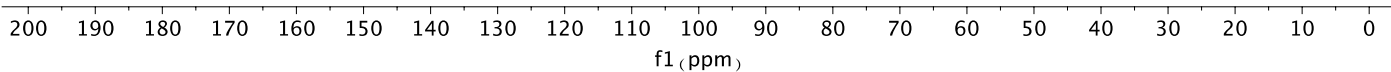



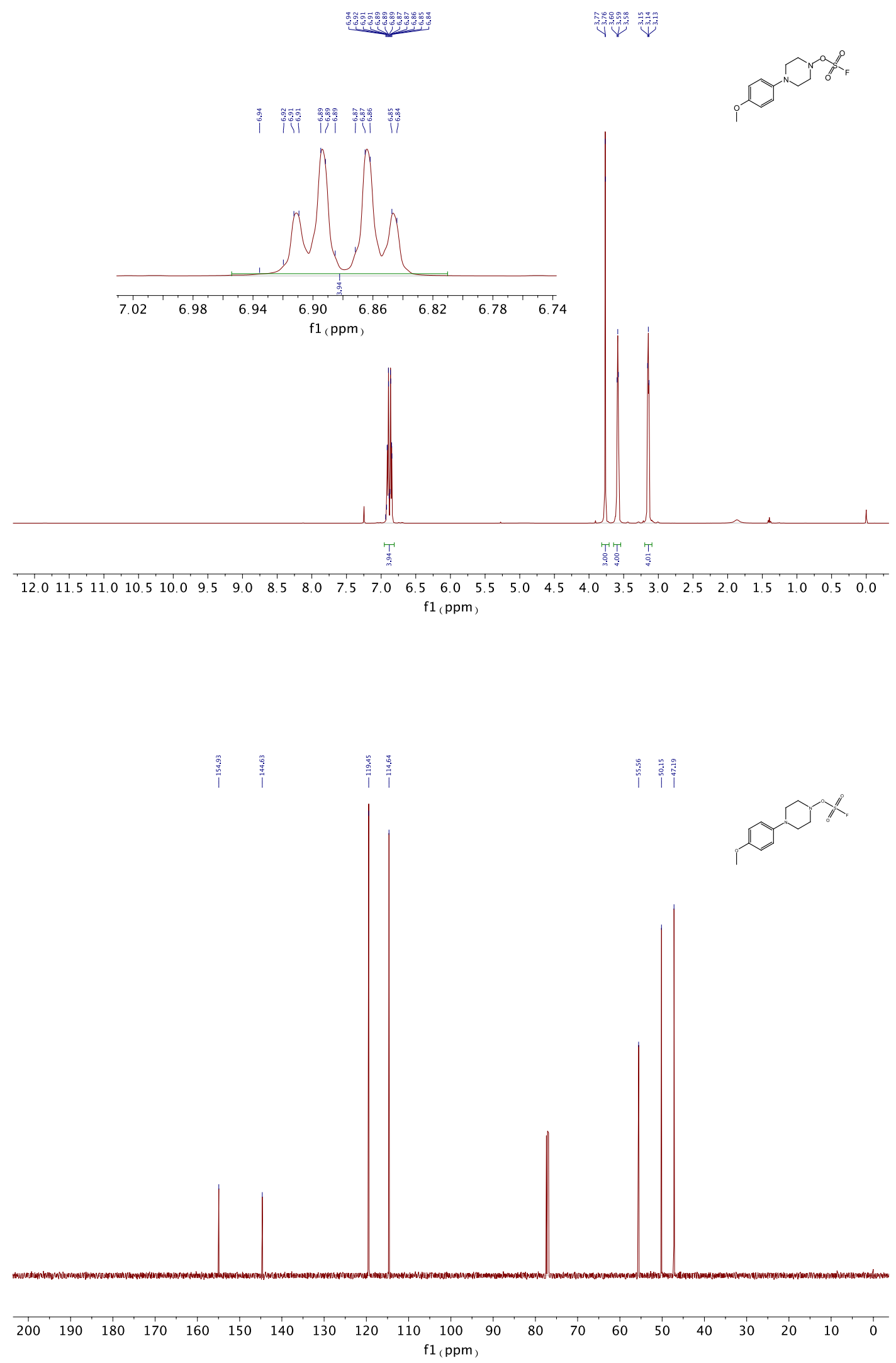
5-methoxy-1H-indole-1-sulfonyl fluoride（1n)
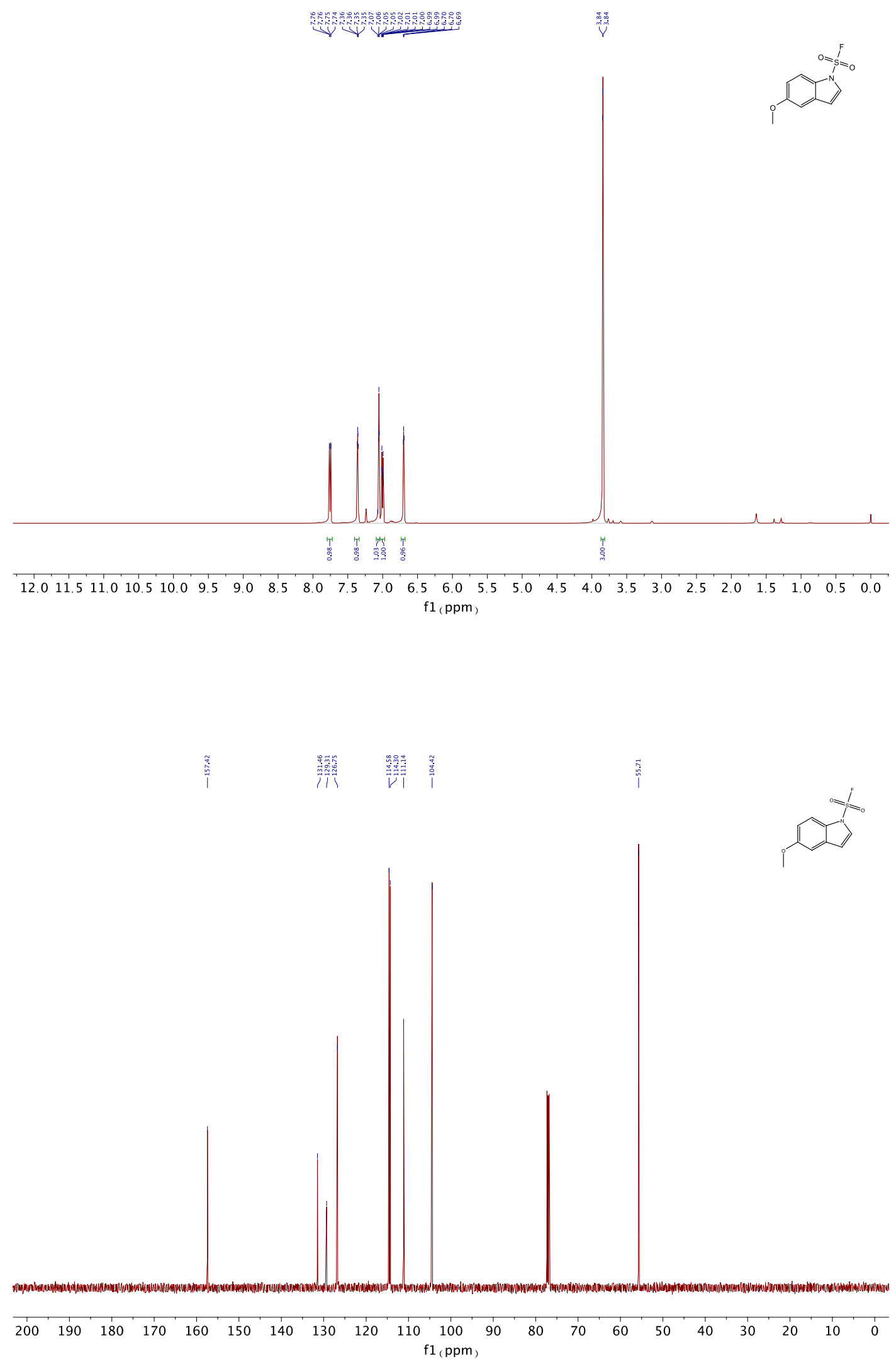
4-(((3R,4R)-4-(3,4-dimethoxybenzyl)-2-oxotetrahydrofuran-3-yl)methyl)-2-methoxyphenyl sulfurofluoridate (1s)

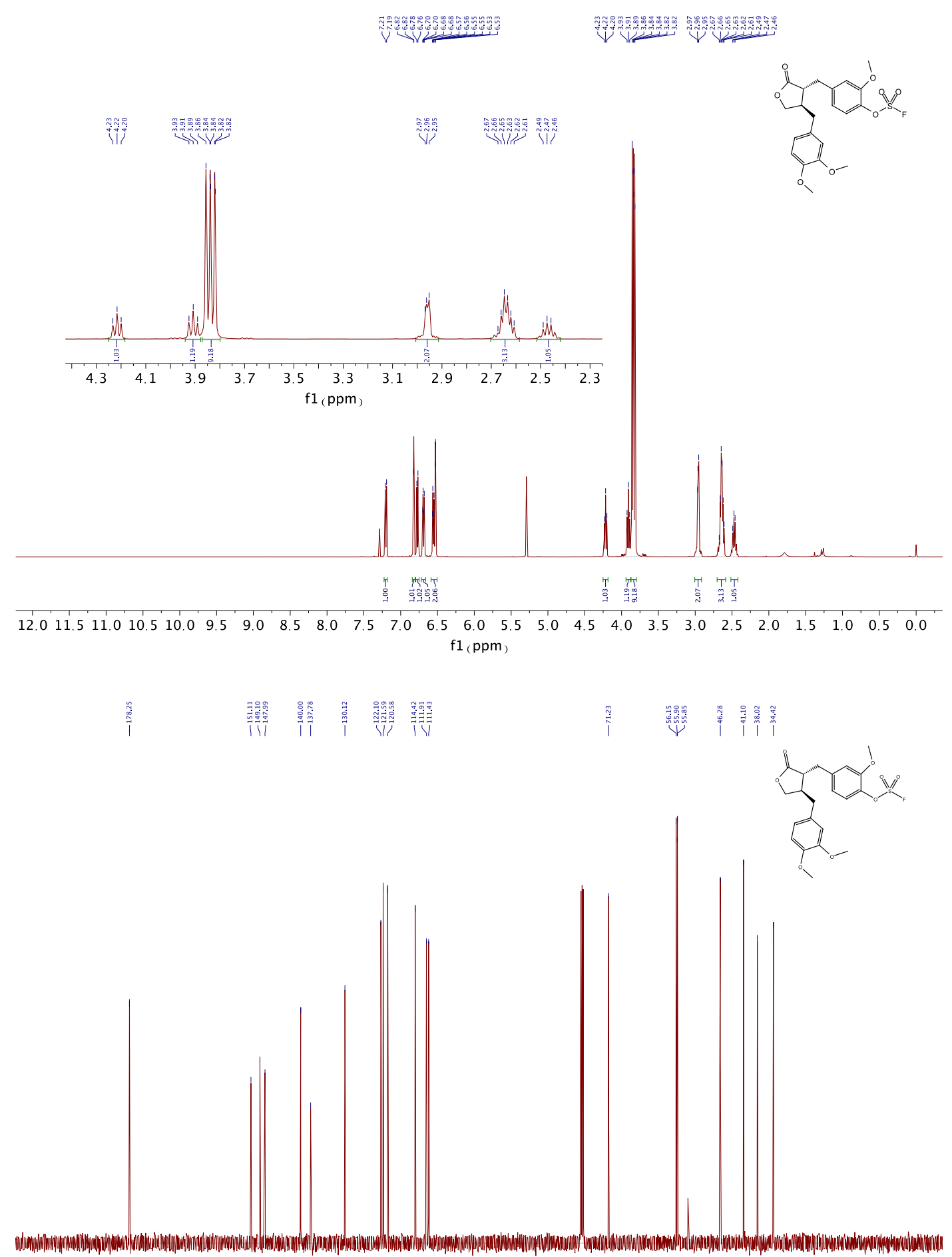

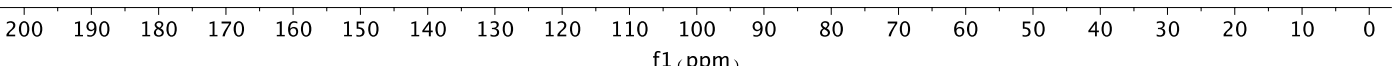


[1,1'-biphenyl]-4-yl benzoylsulfamate (3a1)
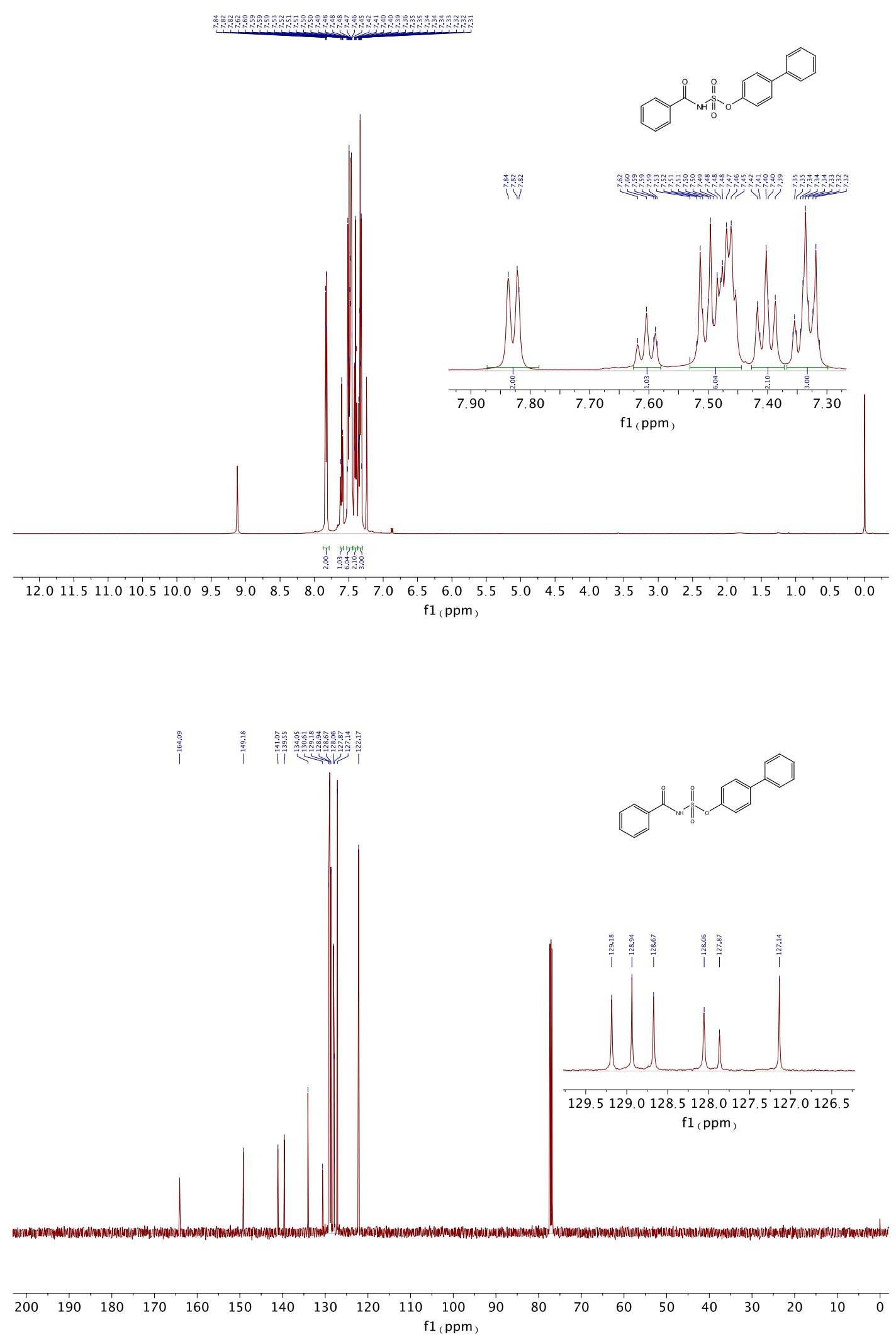

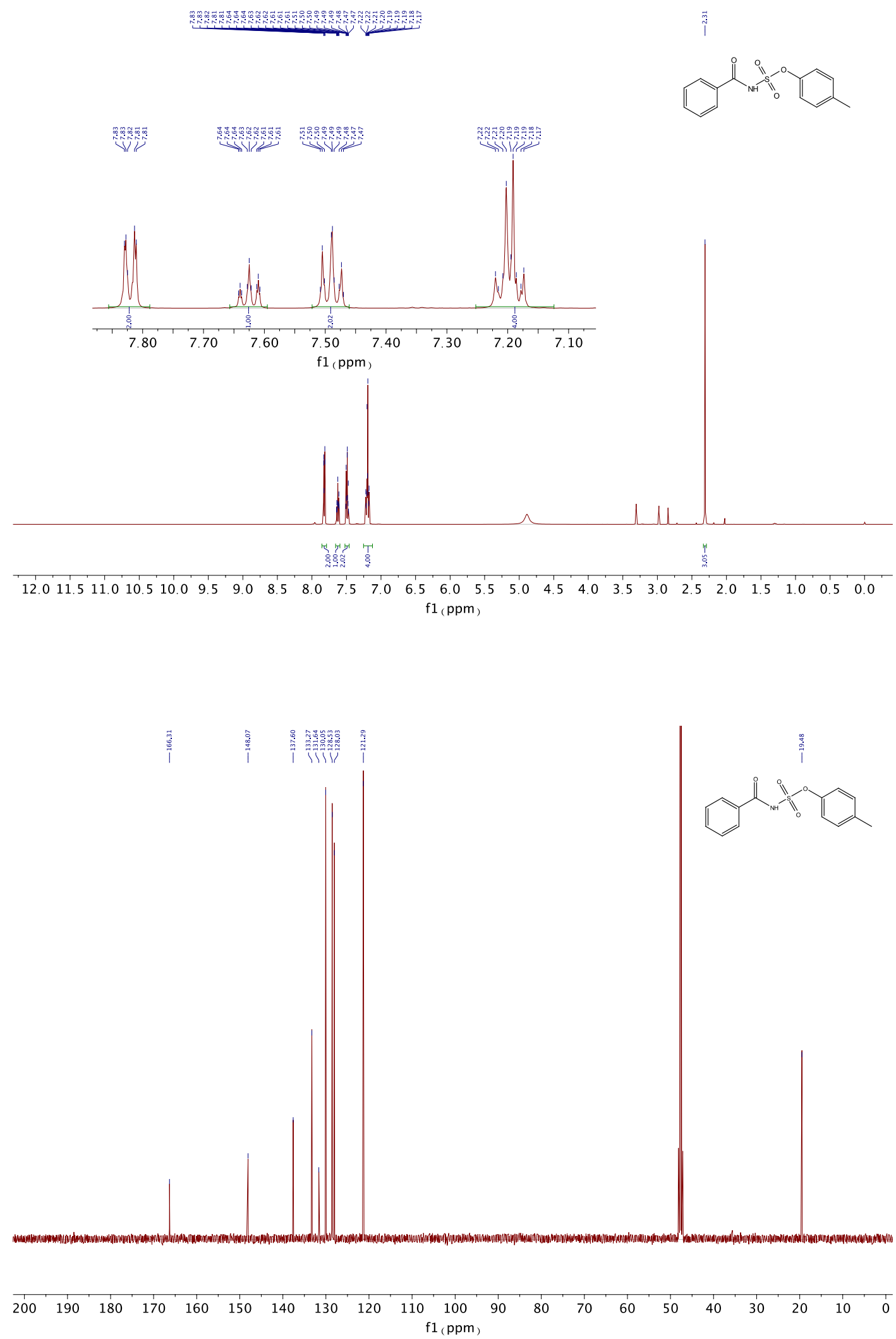
4-methoxyphenyl benzoylsulfamate (3a3)
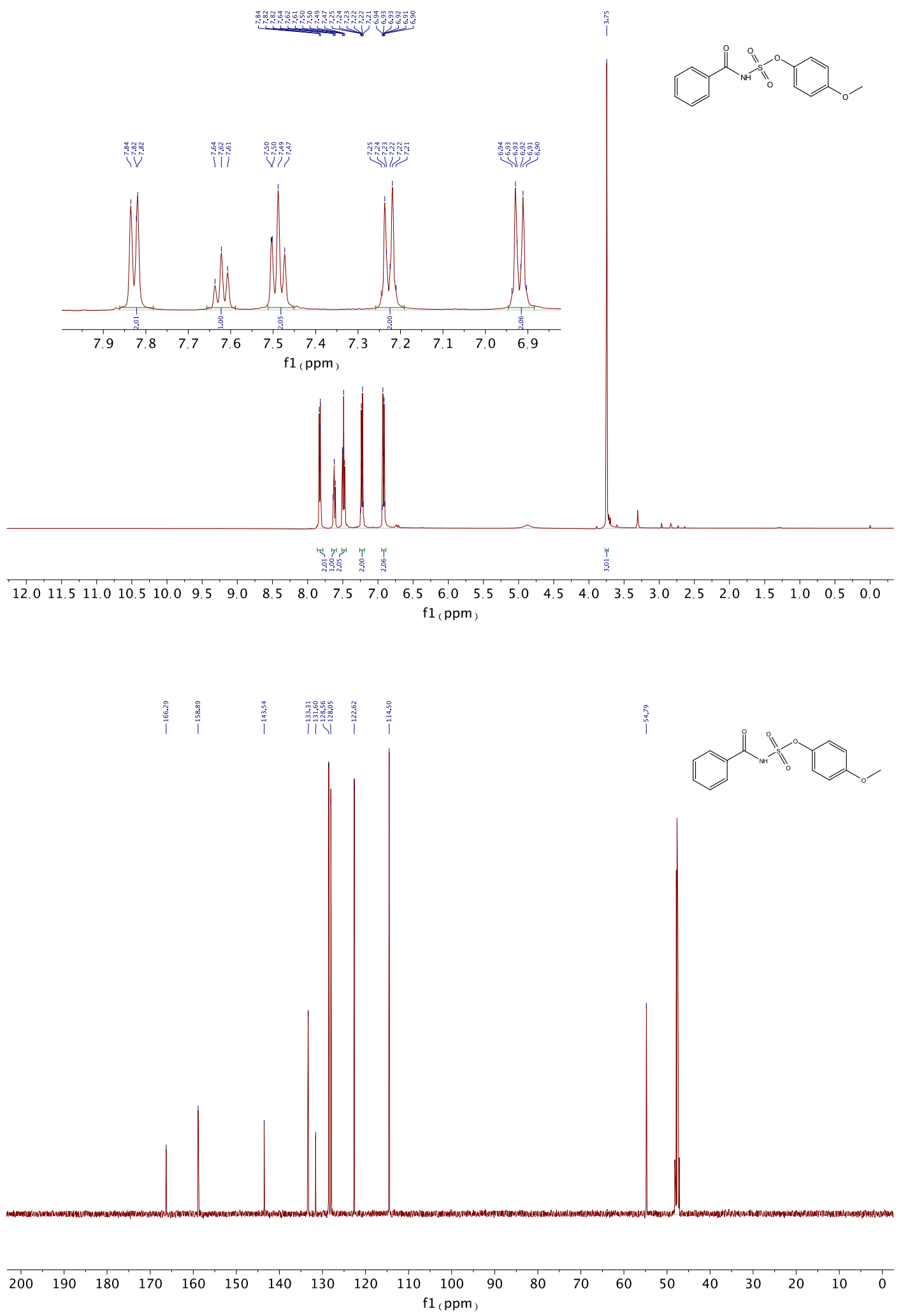

S8 

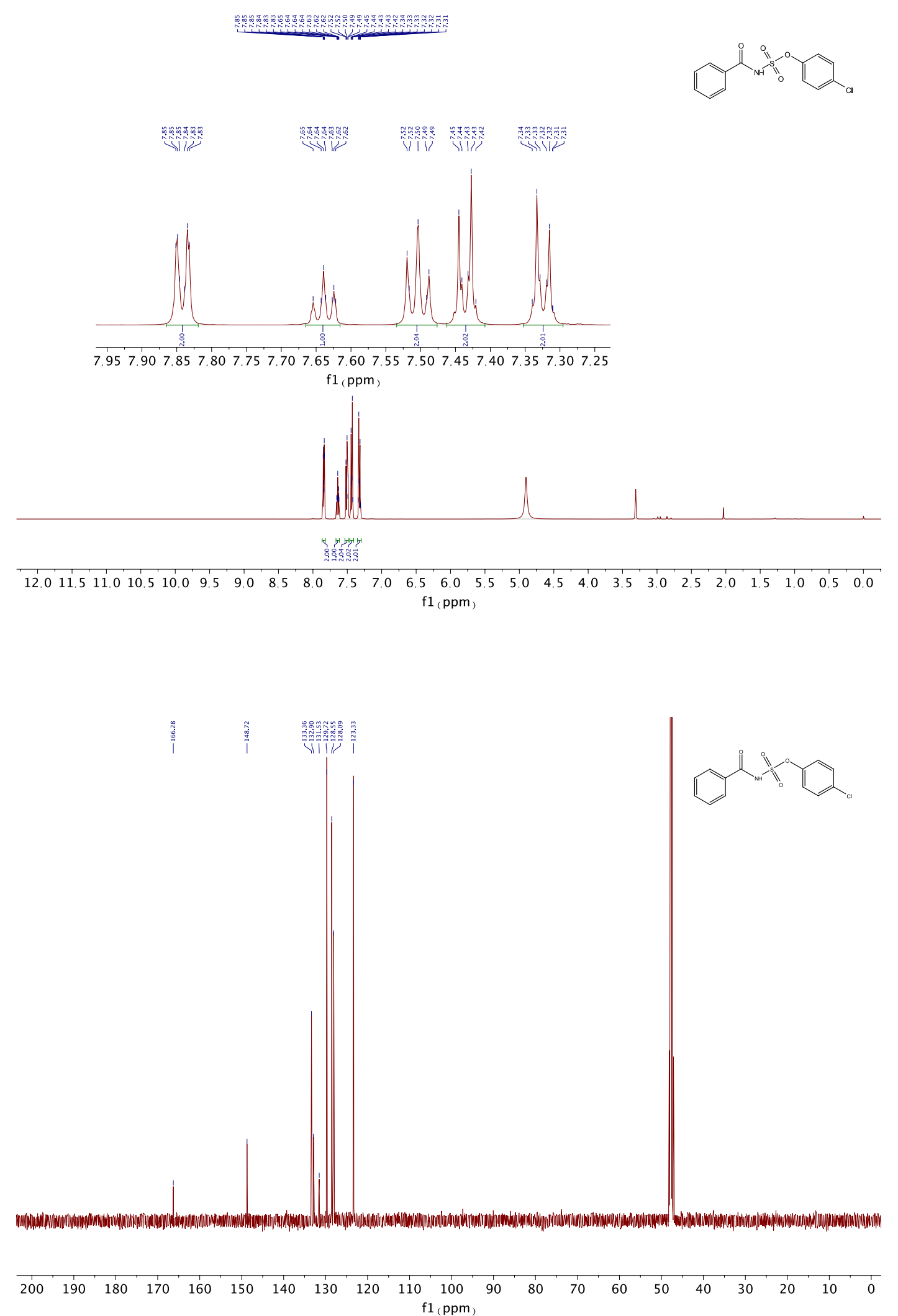

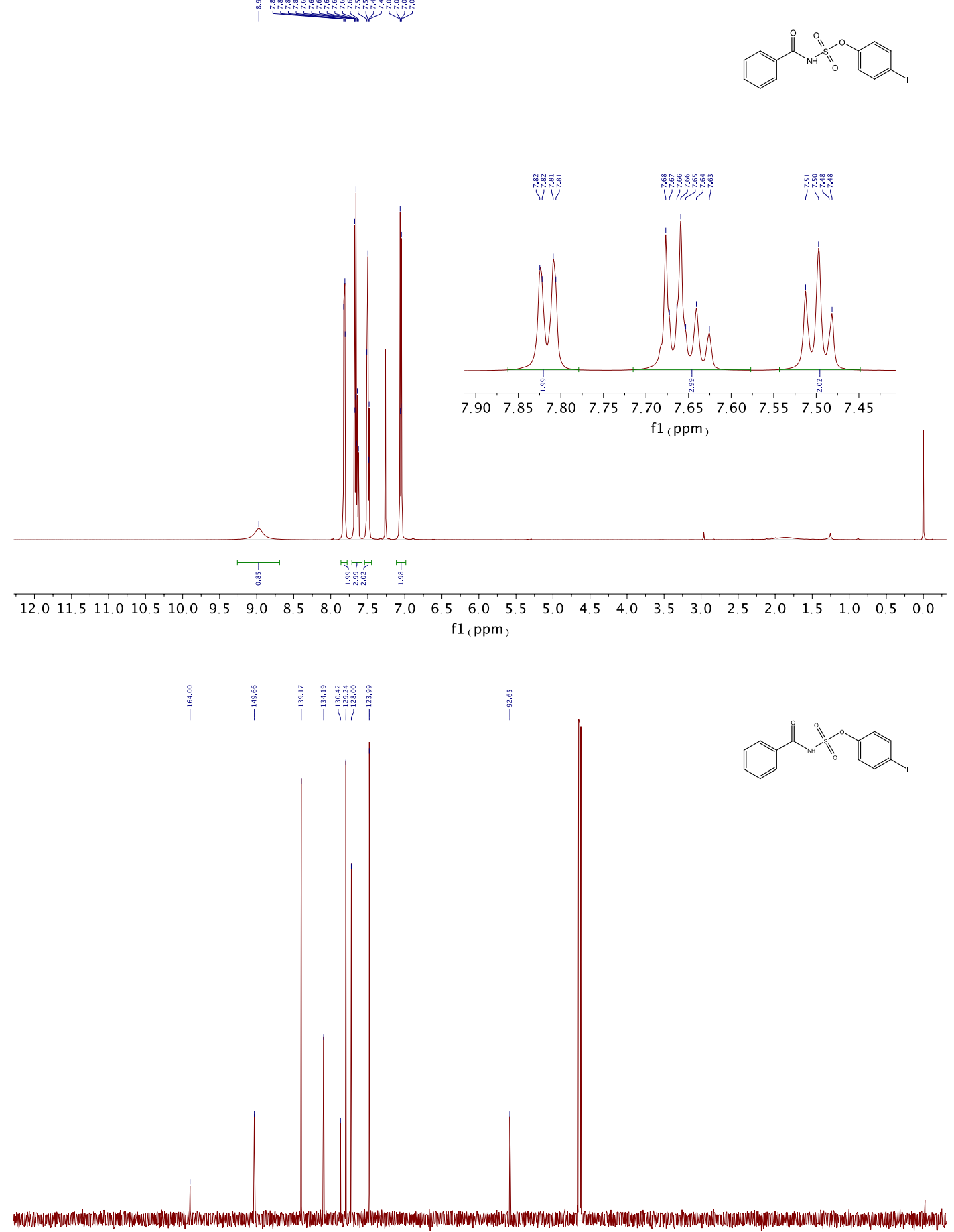

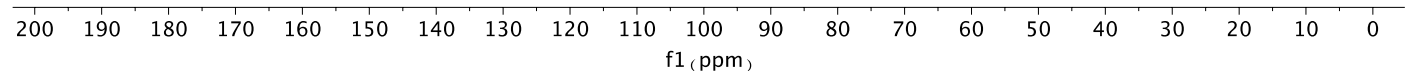




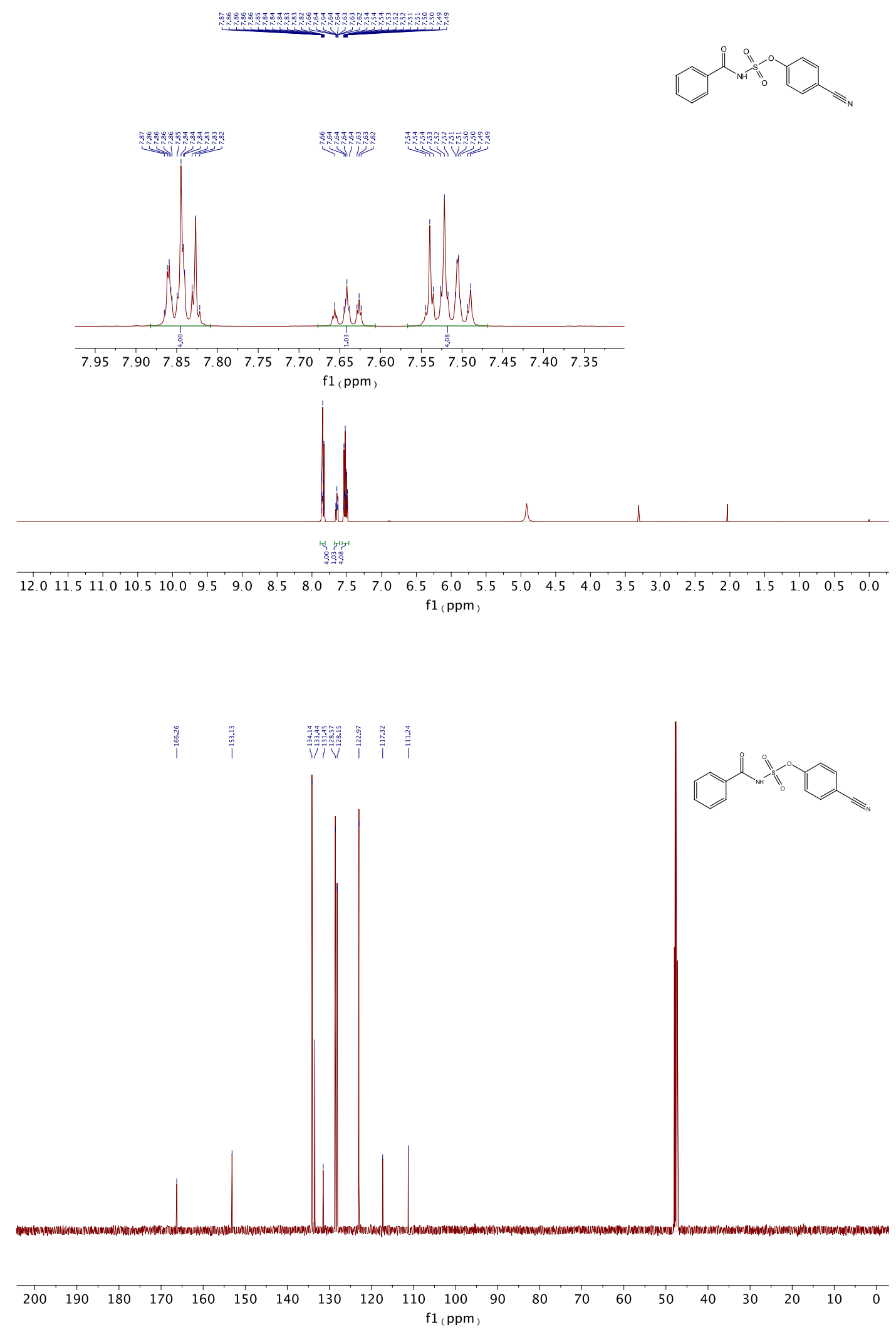



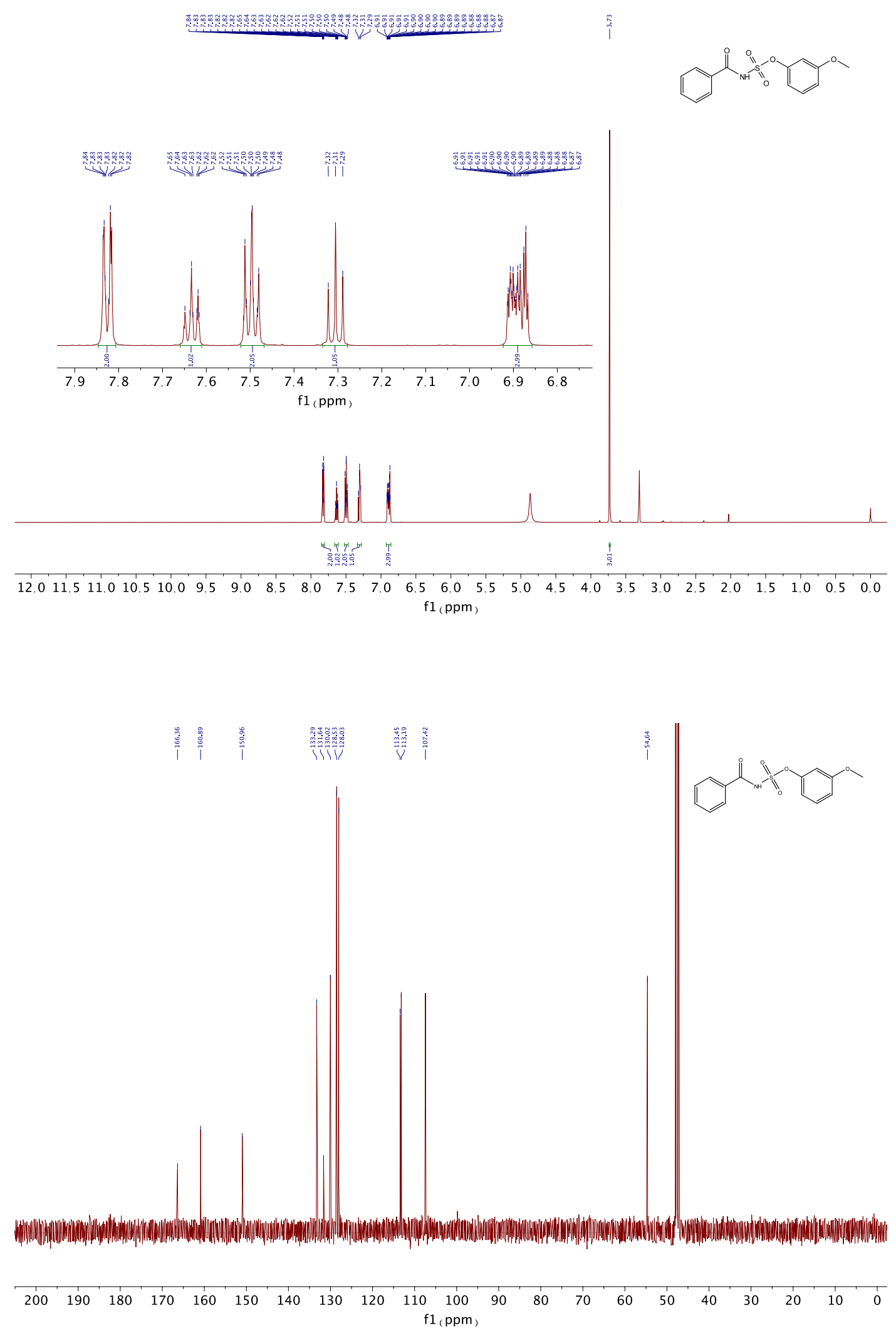

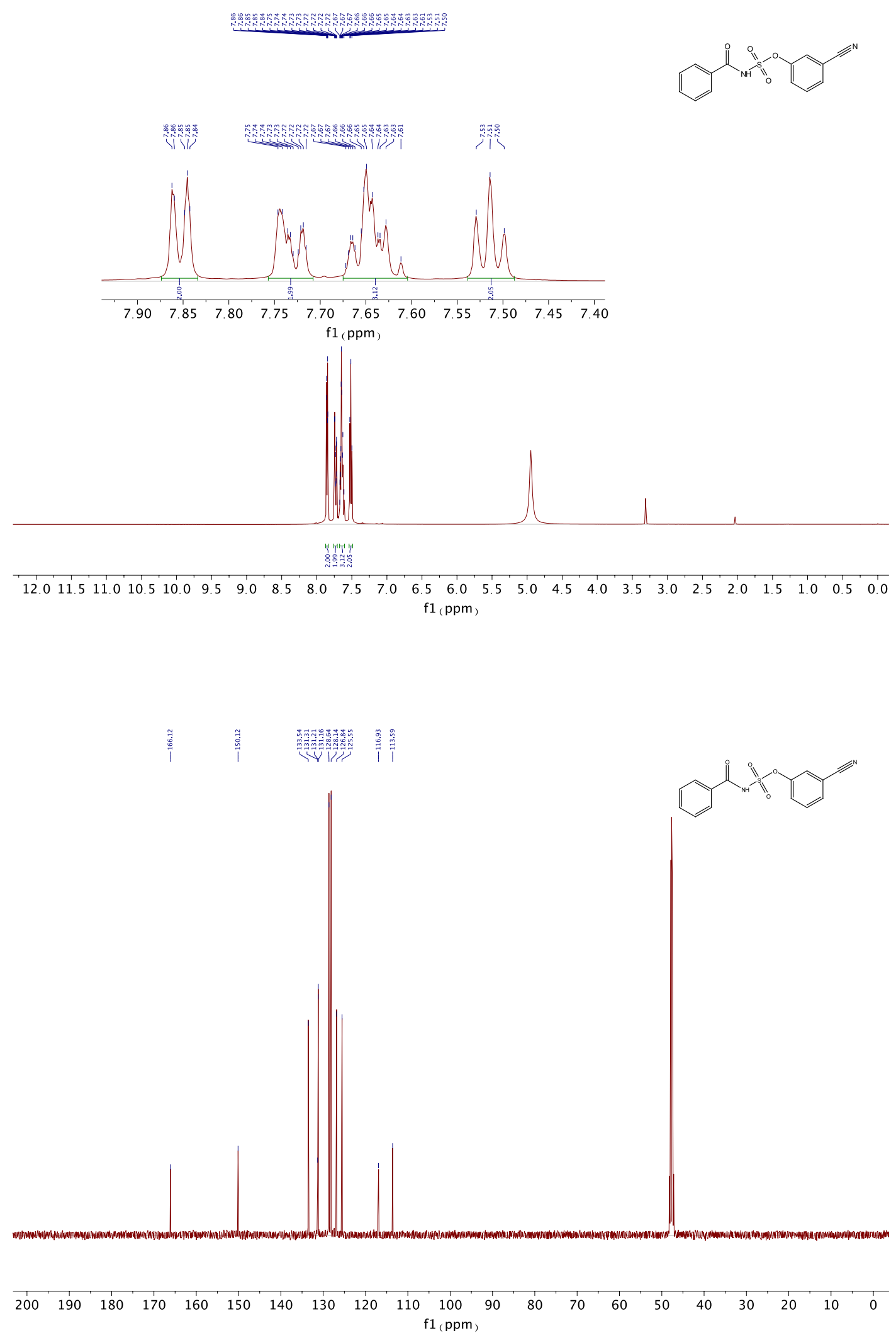


\section{2-(tert-butyl)phenyl benzoylsulfamate (3c1)}

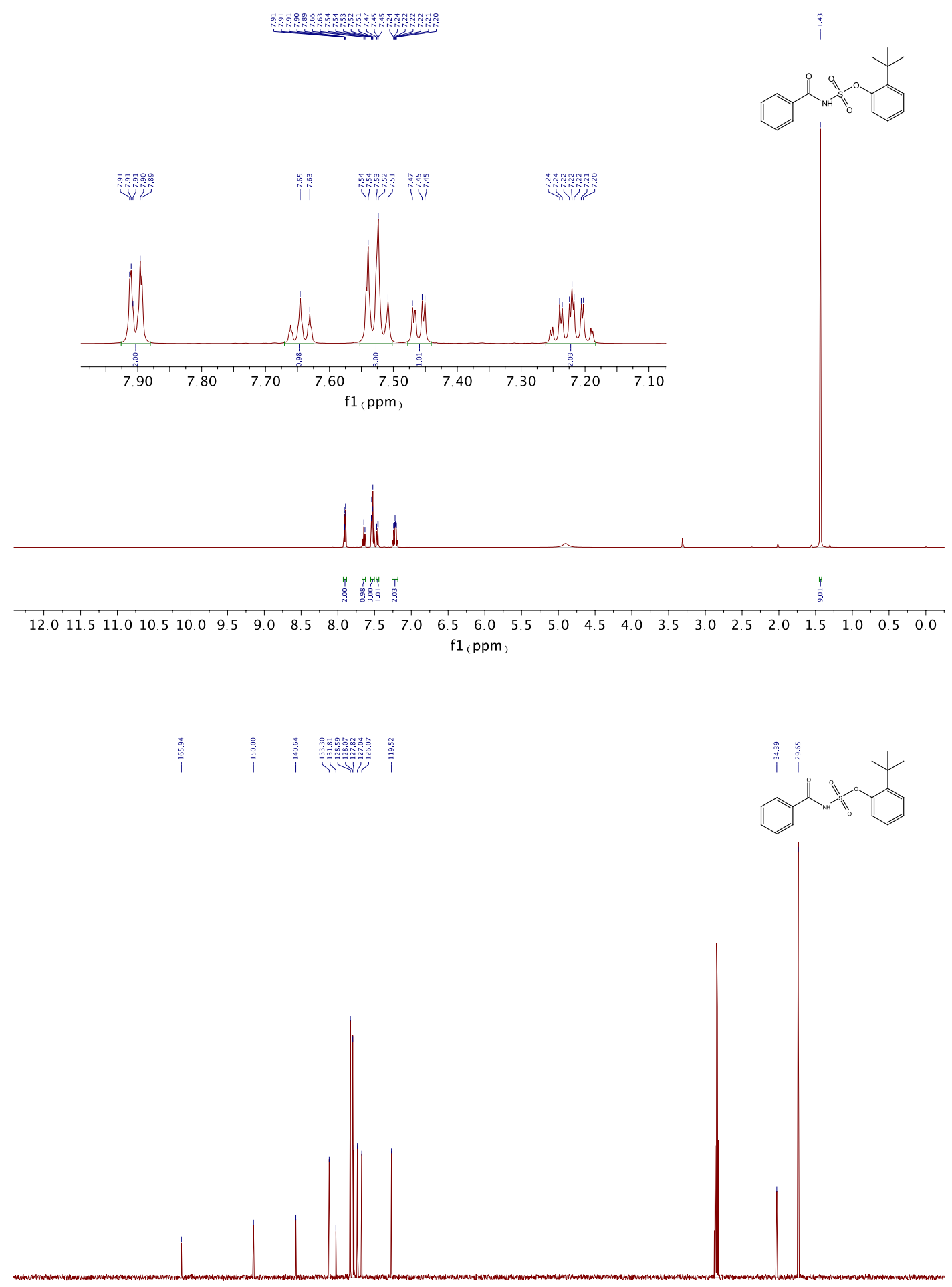

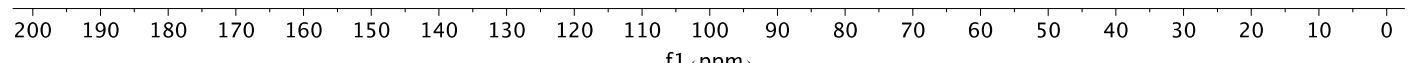




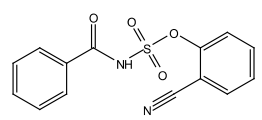

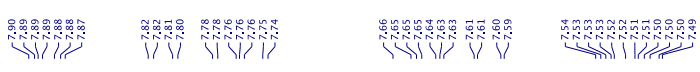
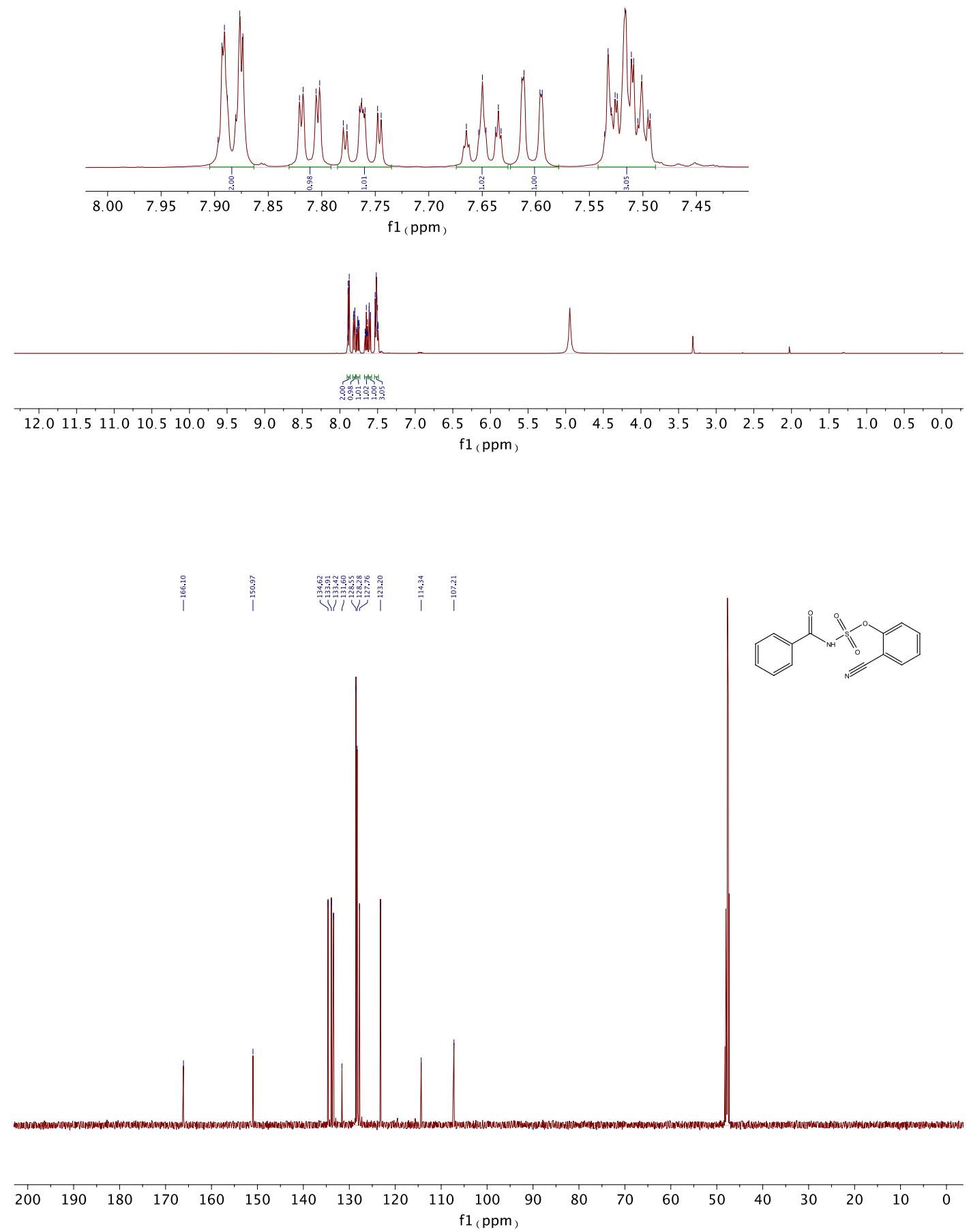


\section{2-bromophenyl benzoylsulfamate (3c3)}
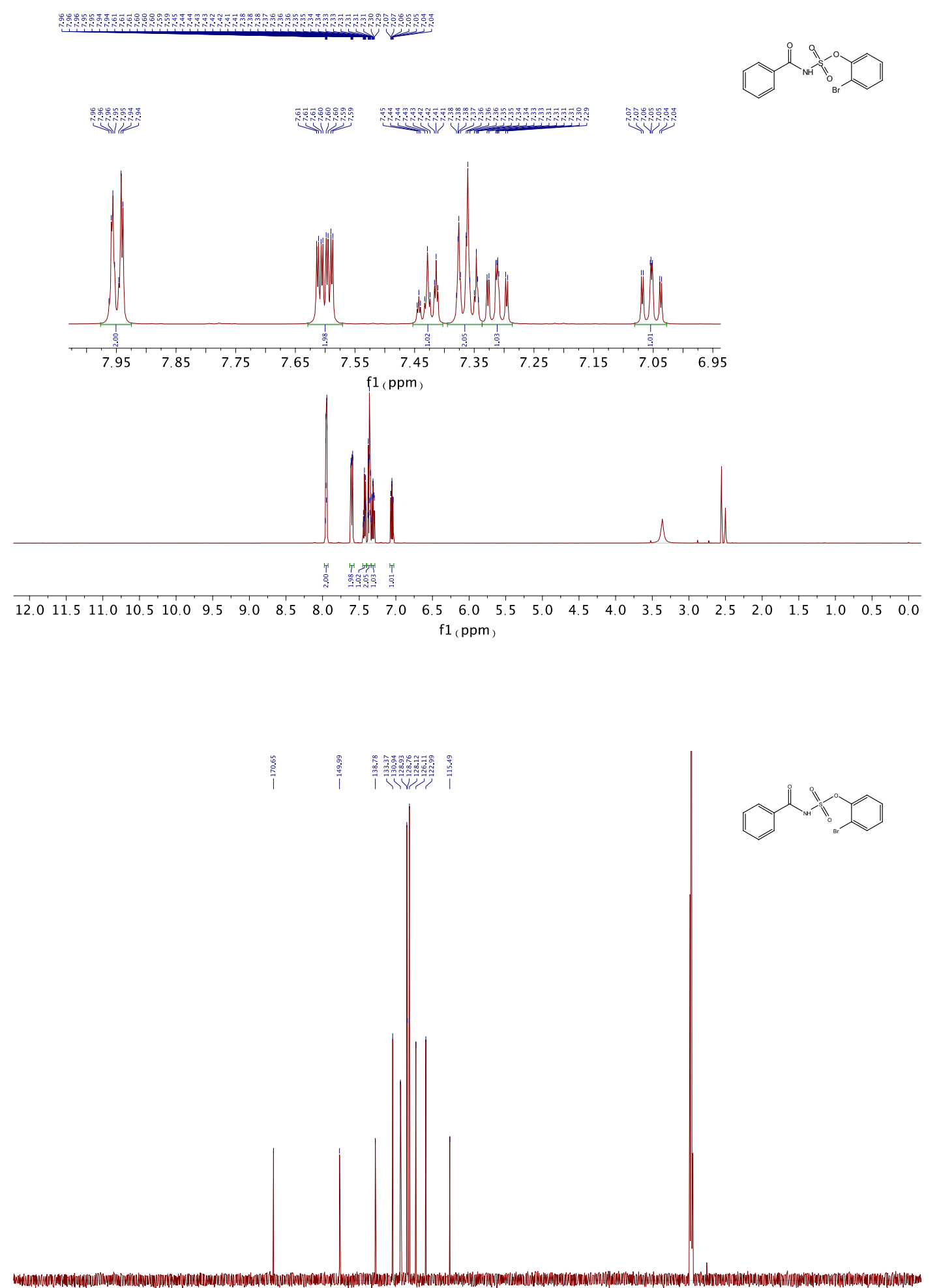

$50 \begin{array}{llllllllllllllllllllllllllllllllll}540 & 230 & 220 & 210 & 200 & 190 & 180 & 170 & 160 & 150 & 140 & 130 & 120 & 110 & 100 & 90 & 80 & 70 & 60 & 50 & 40 & 30 & 20 & 10 & 0 & 10 & 20 & -31\end{array}$

$\mathrm{f} 1(\mathrm{ppm})$ 
2,6-dimethylphenyl benzoylsulfamate (3d)

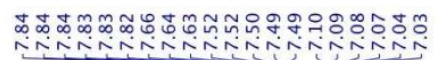

$\stackrel{\hat{m}}{i}$
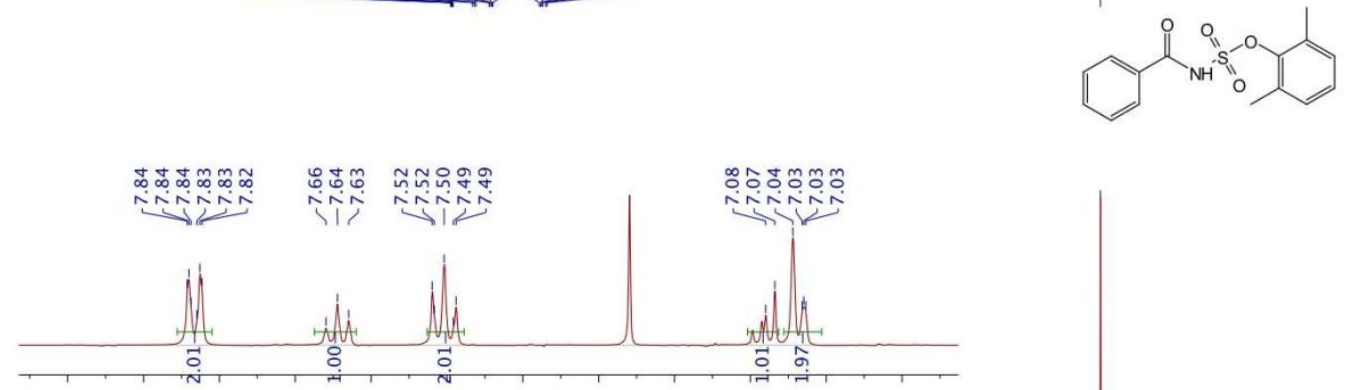

8.07 .97 .87 .77 .67 .57 .47 .37 .27 .17 .06 .9 $\mathrm{f1}(\mathrm{ppm})$
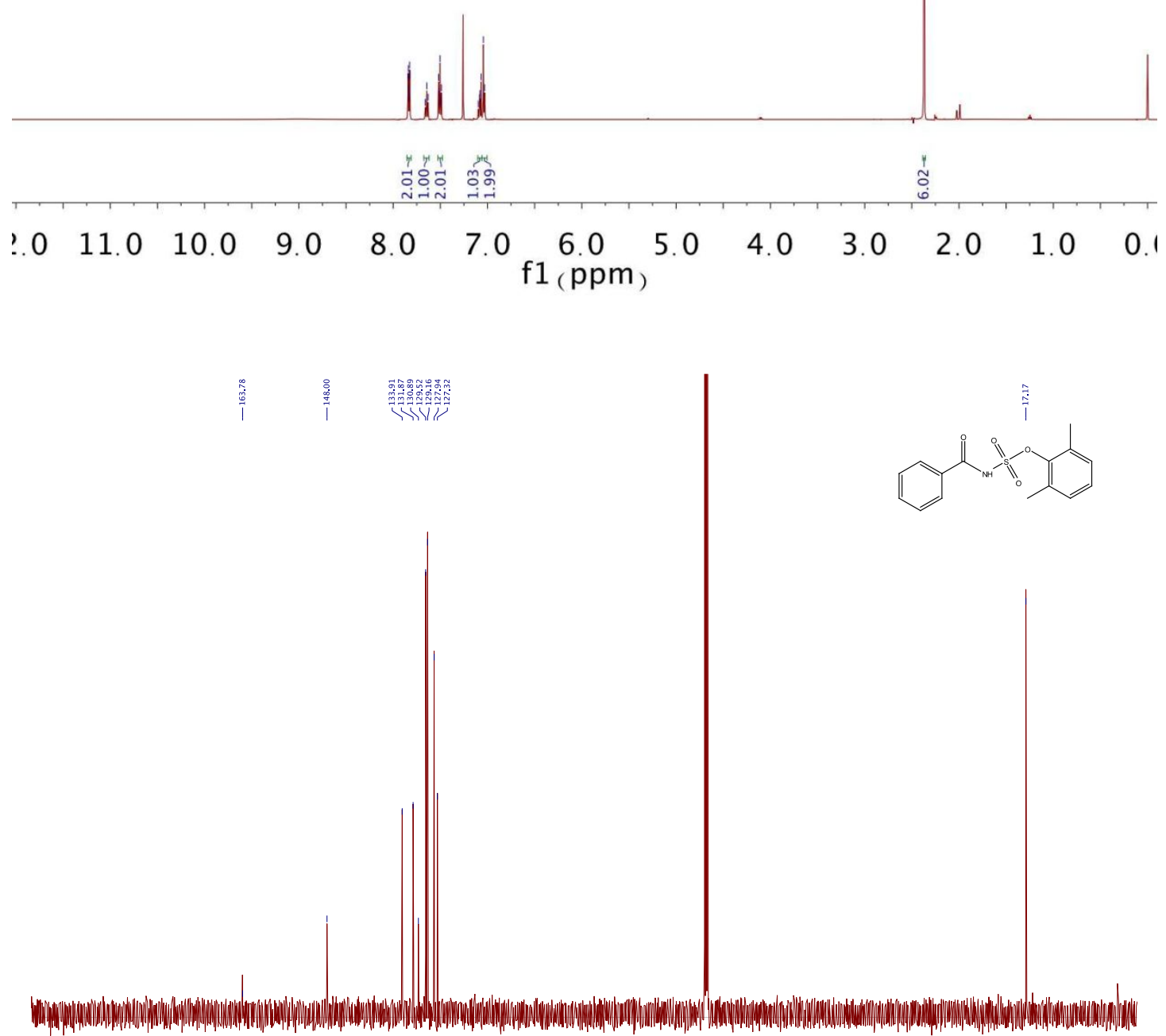

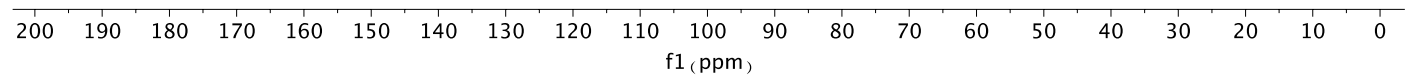


pyridin-3-yl benzoylsulfamate (3e)

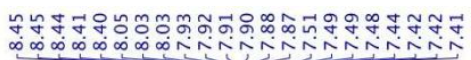

$\overbrace{}^{2}$

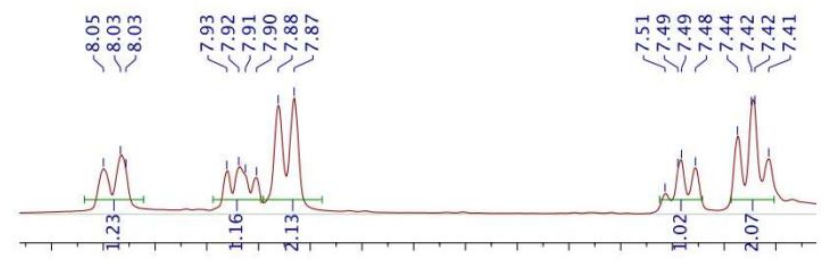

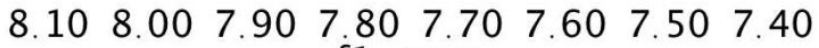

f1 ( $\mathrm{ppm})$

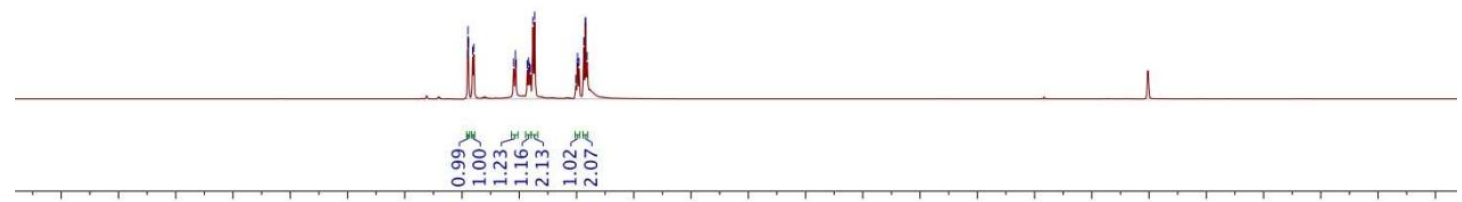

$\begin{array}{llllllllllll}12.0 & 11.0 & 10.0 & 9.0 & 8.0 & \begin{array}{l}7.0 \\ \mathrm{f} 1 \underset{(\mathrm{ppm})}{6.0}\end{array} & 5.0 & 4.0 & 3.0 & 2.0 & 1.0 & 0 . \mathrm{C}\end{array}$

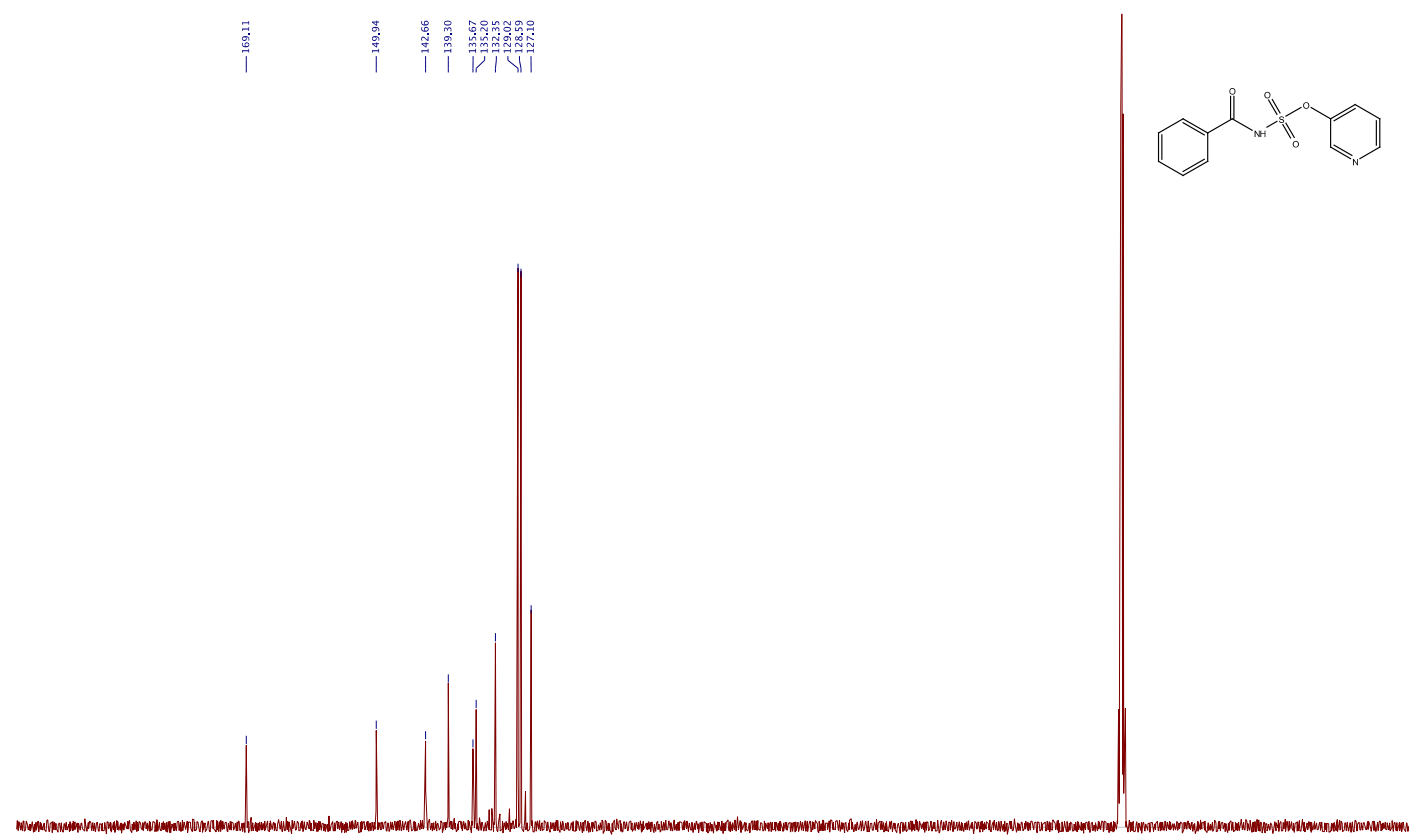

\begin{tabular}{lllllllllllllllllllll}
\hline 200 & 190 & 180 & 170 & 160 & 150 & 140 & 130 & 120 & 110 & 100 & 90 & 80 & 70 & 60 & 50 & 40 & 30 & 20 & 10 & 0
\end{tabular} 
quinolin-8-yl benzoylsulfamate (3f)

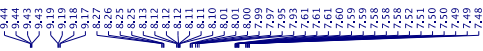
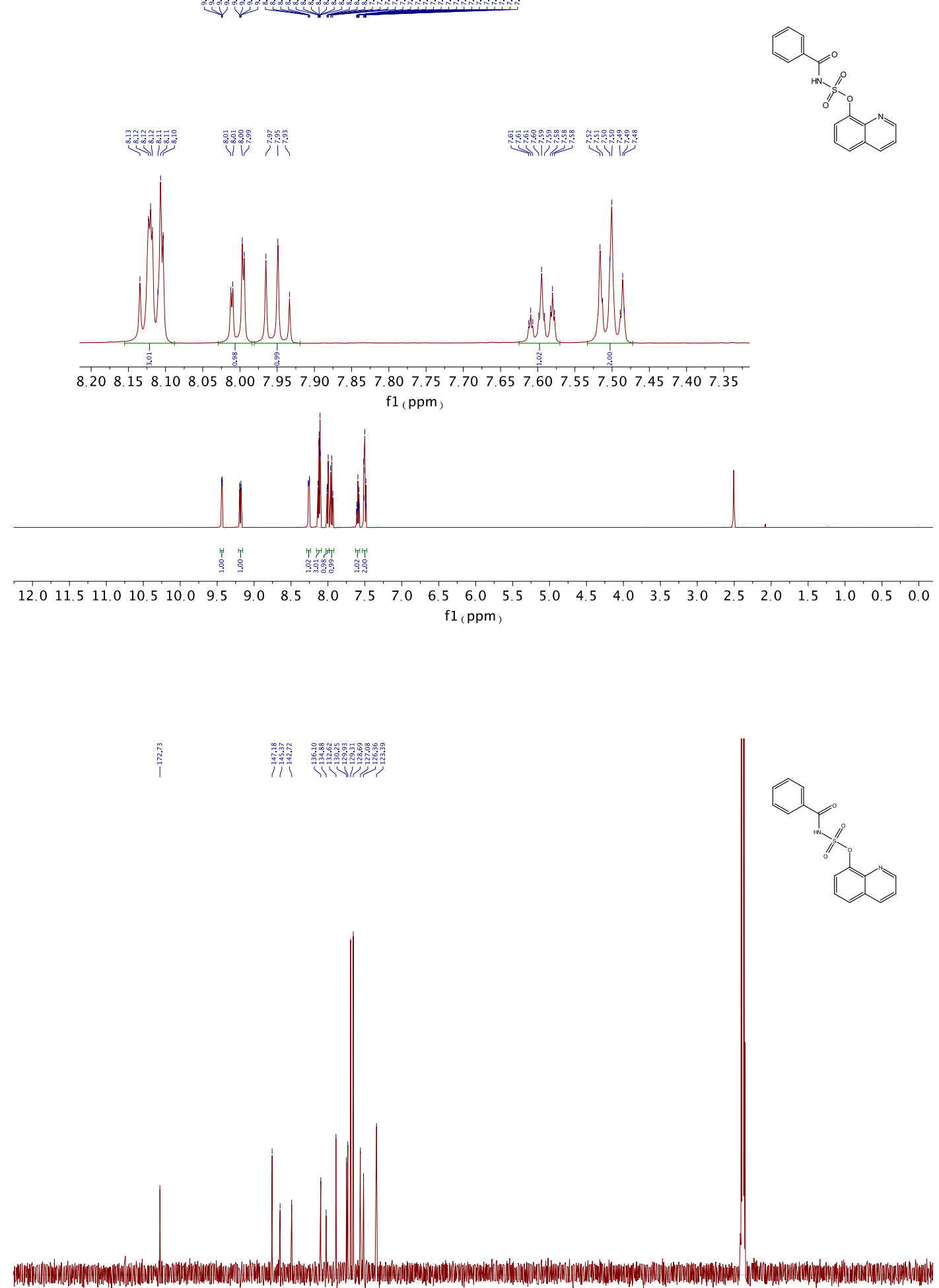

$\begin{array}{llllllllll}190 & 180 & 170 & 160 & 150 & 140 & 130 & 120 & 110 & 100\end{array}$

$\mathrm{f1}$ ( $\mathrm{ppm}$ ) 
p-tolyl (4-methoxybenzoyl)sulfamate (3g)

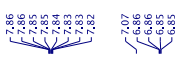

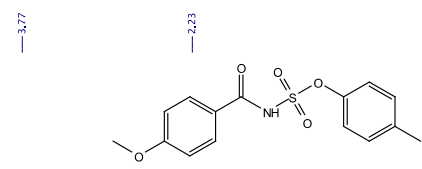

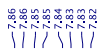
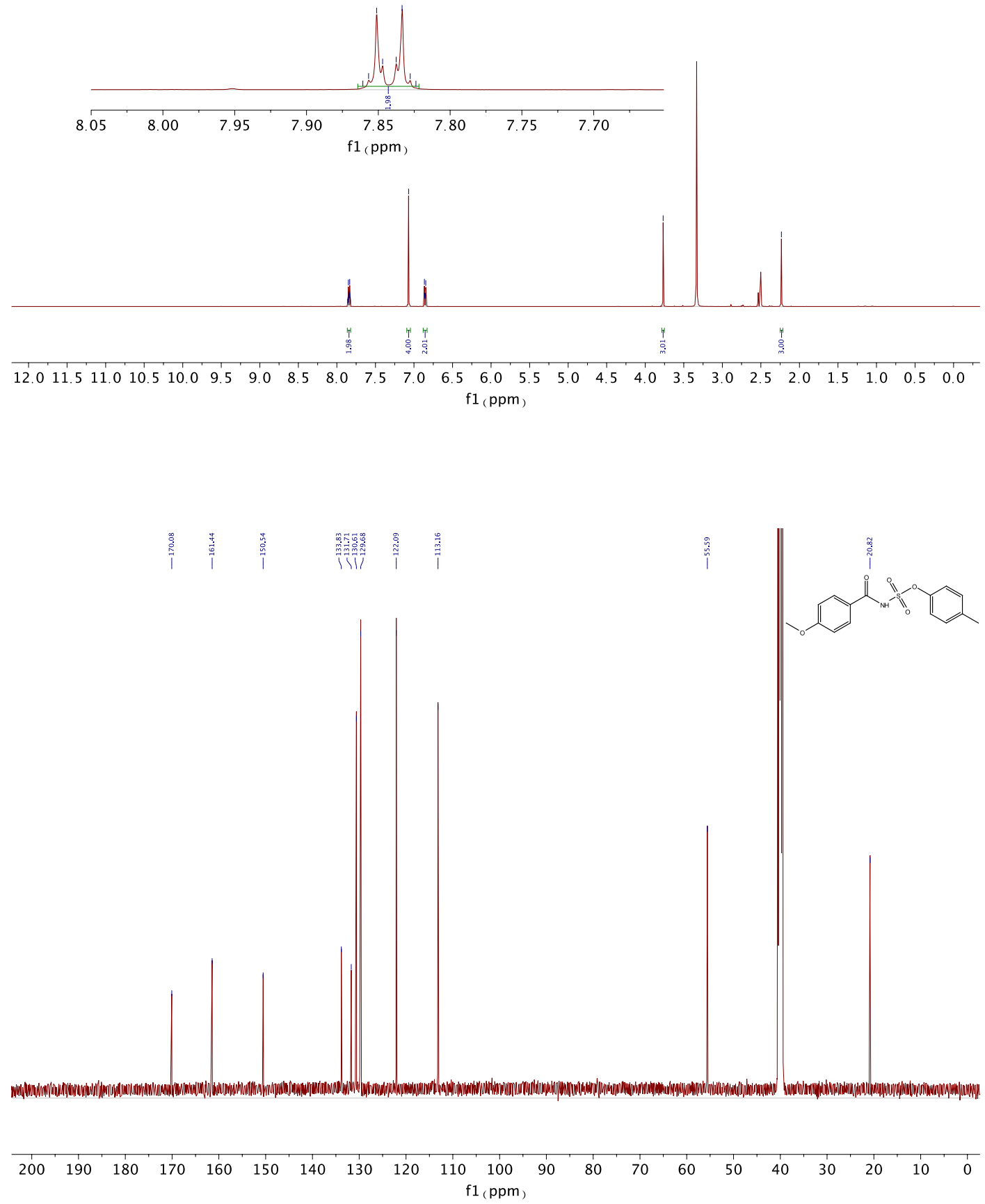
p-tolyl (4-bromobenzoyl)sulfamate (3h)

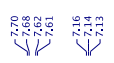
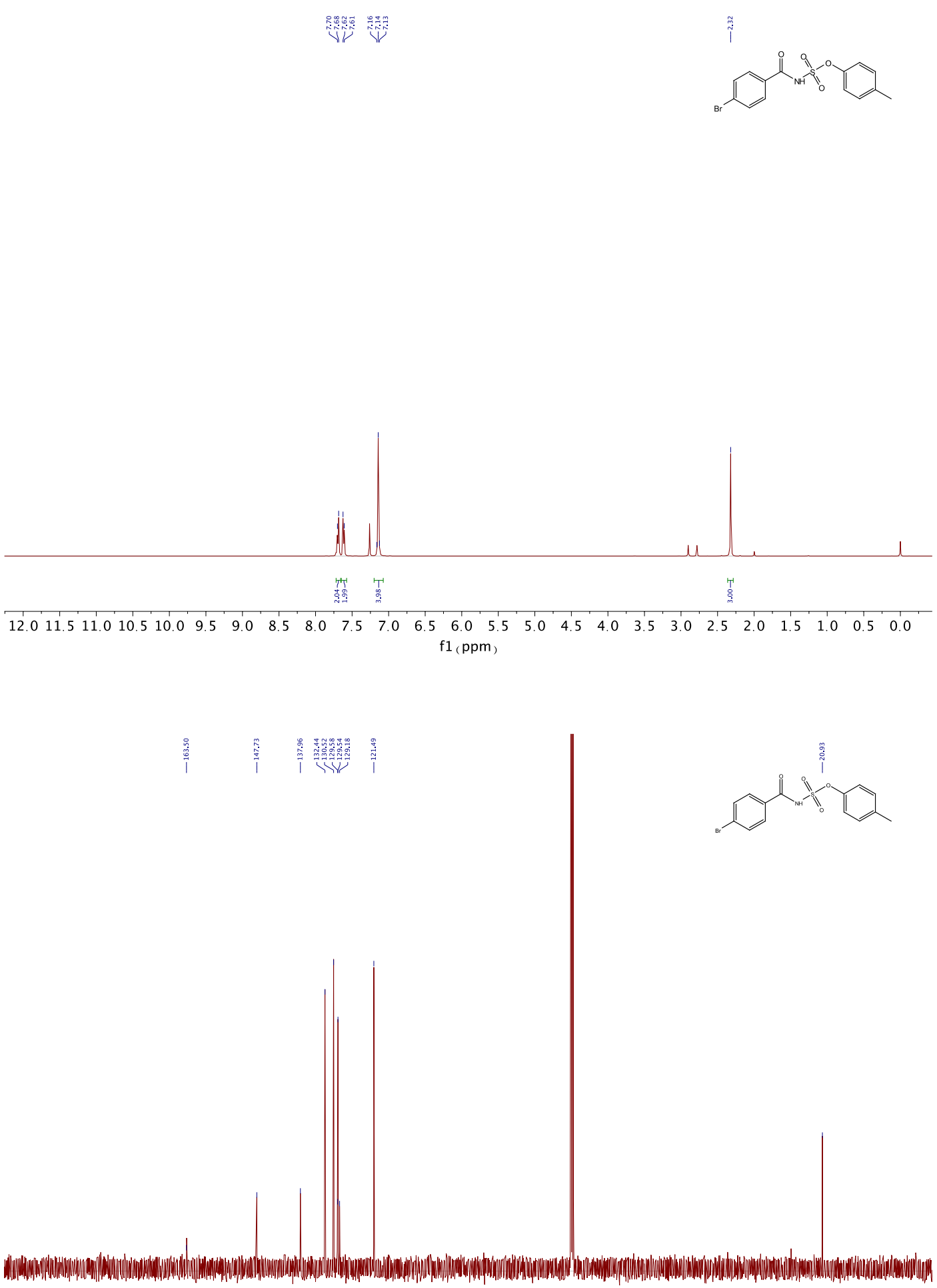

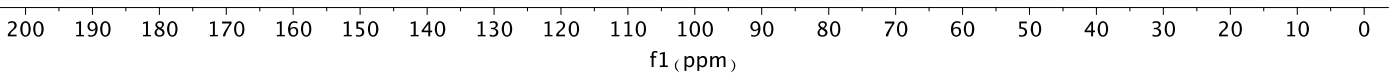

S2 
p-tolyl (4-(trifluoromethyl)benzoyl)sulfamate (3i)

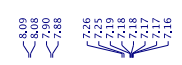
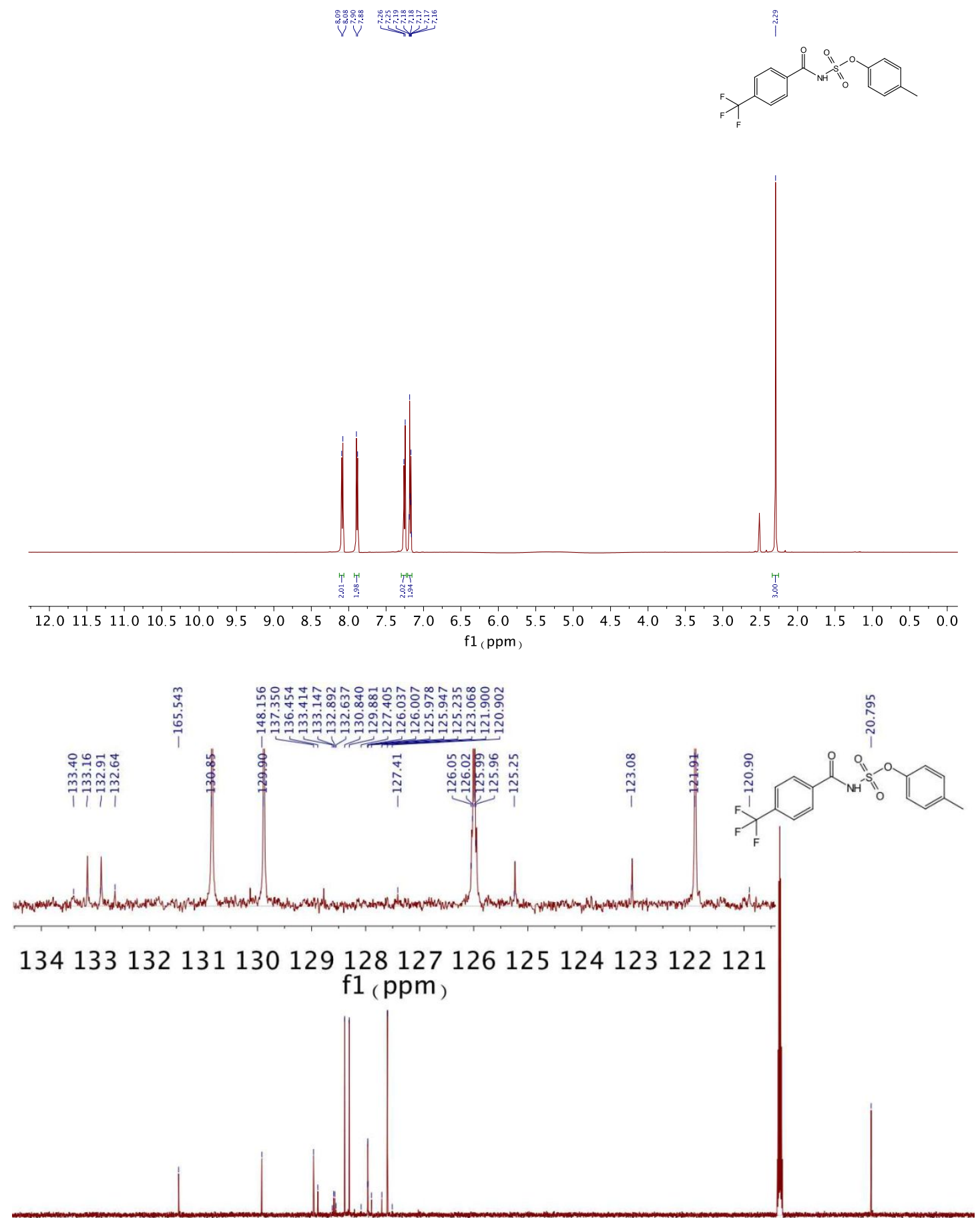

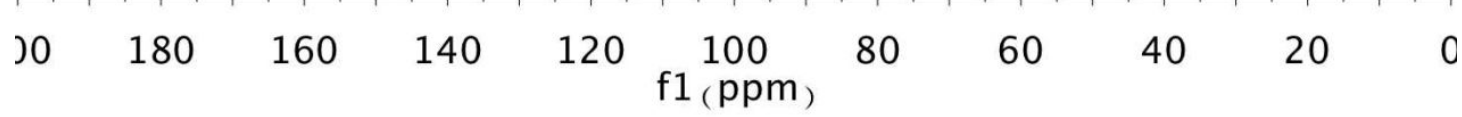


p-tolyl benzoylsulfamate (3j)

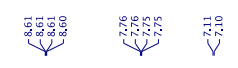
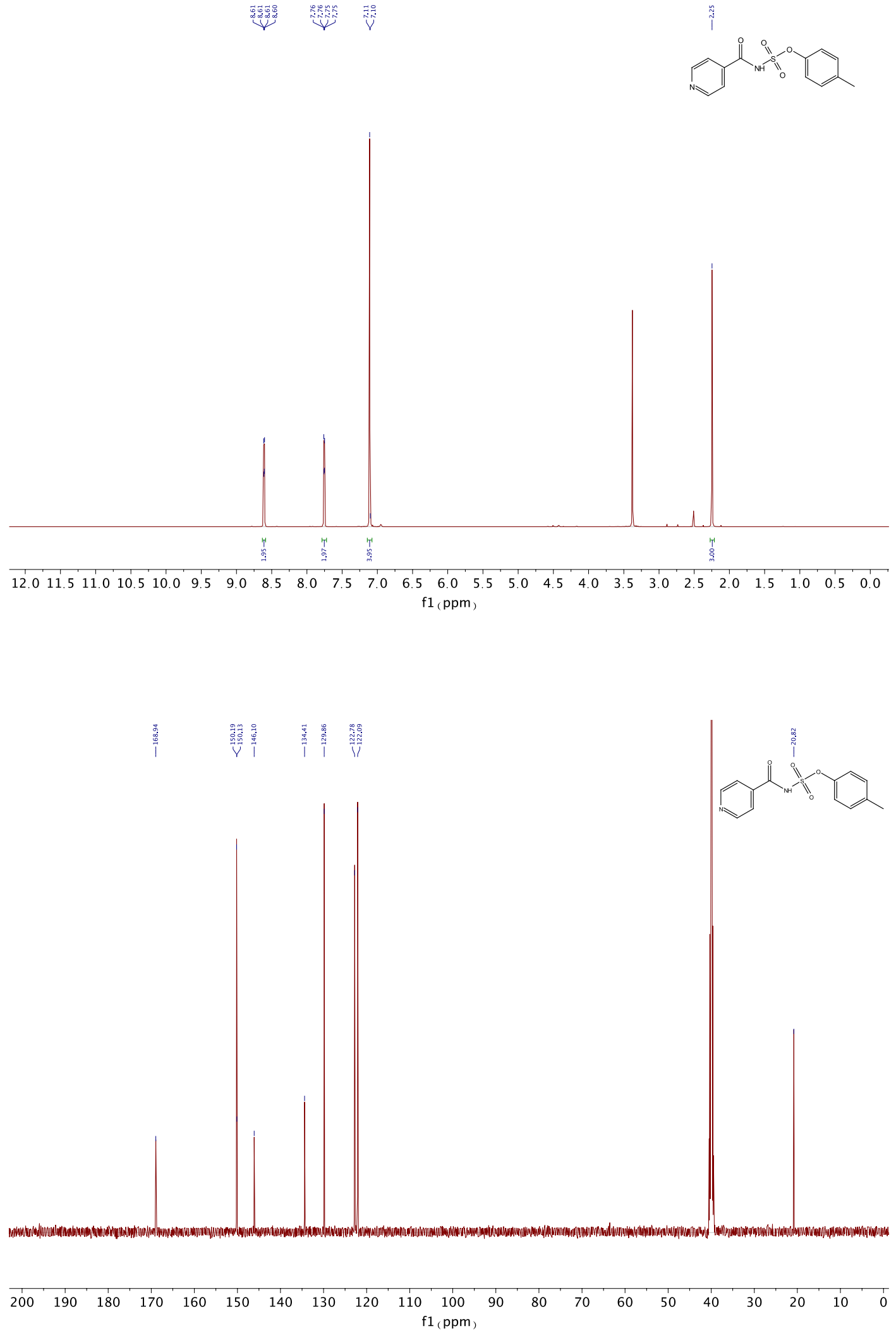

S2. 
p-tolyl (thiophene-2-carbonyl)sulfamate (3k)
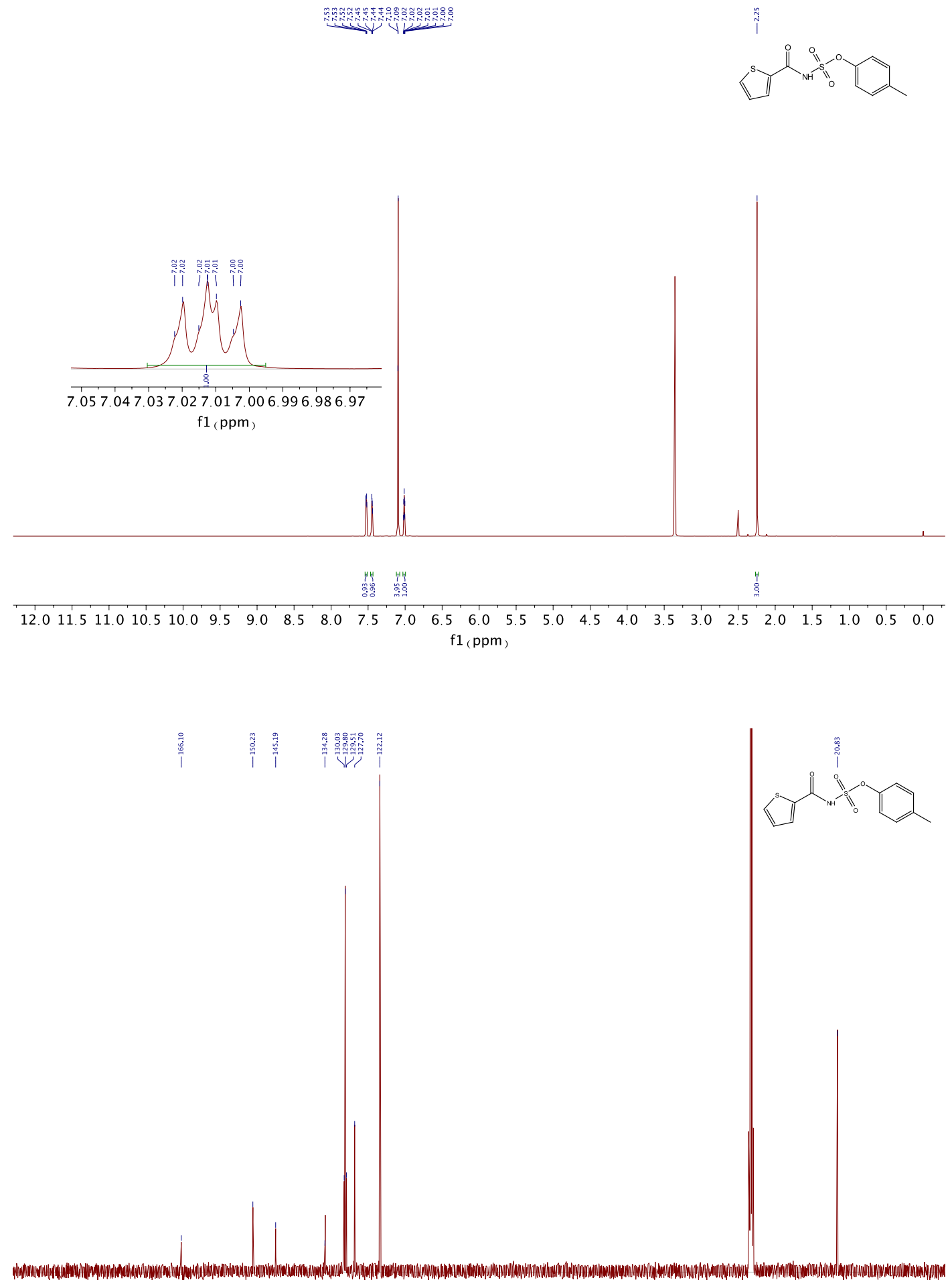

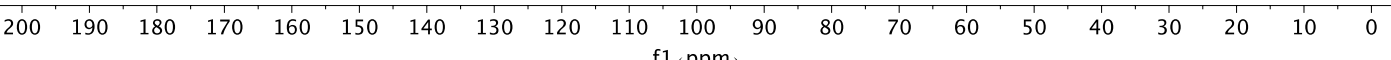


p-tolyl tridecanoylsulfamate (3I)
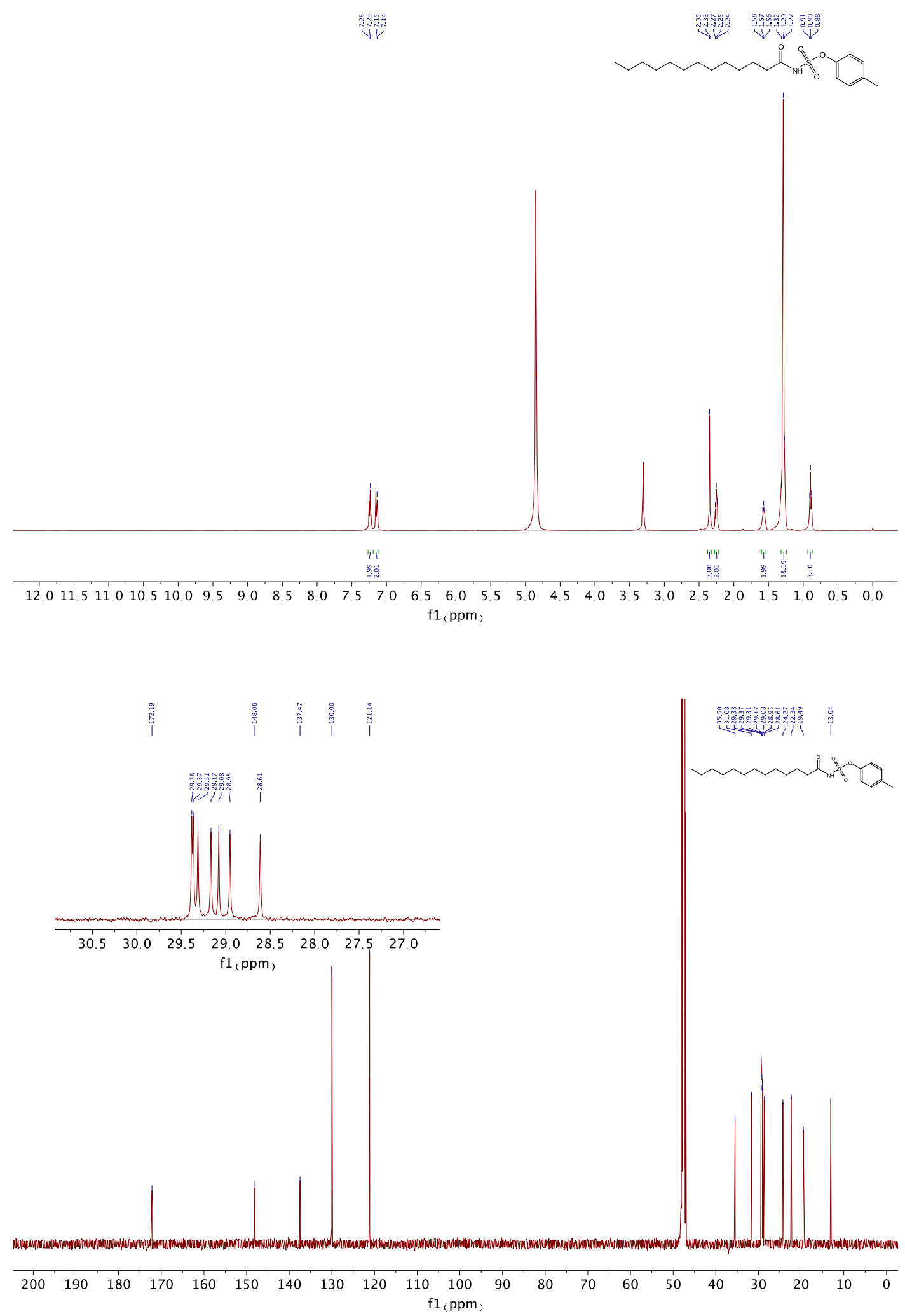


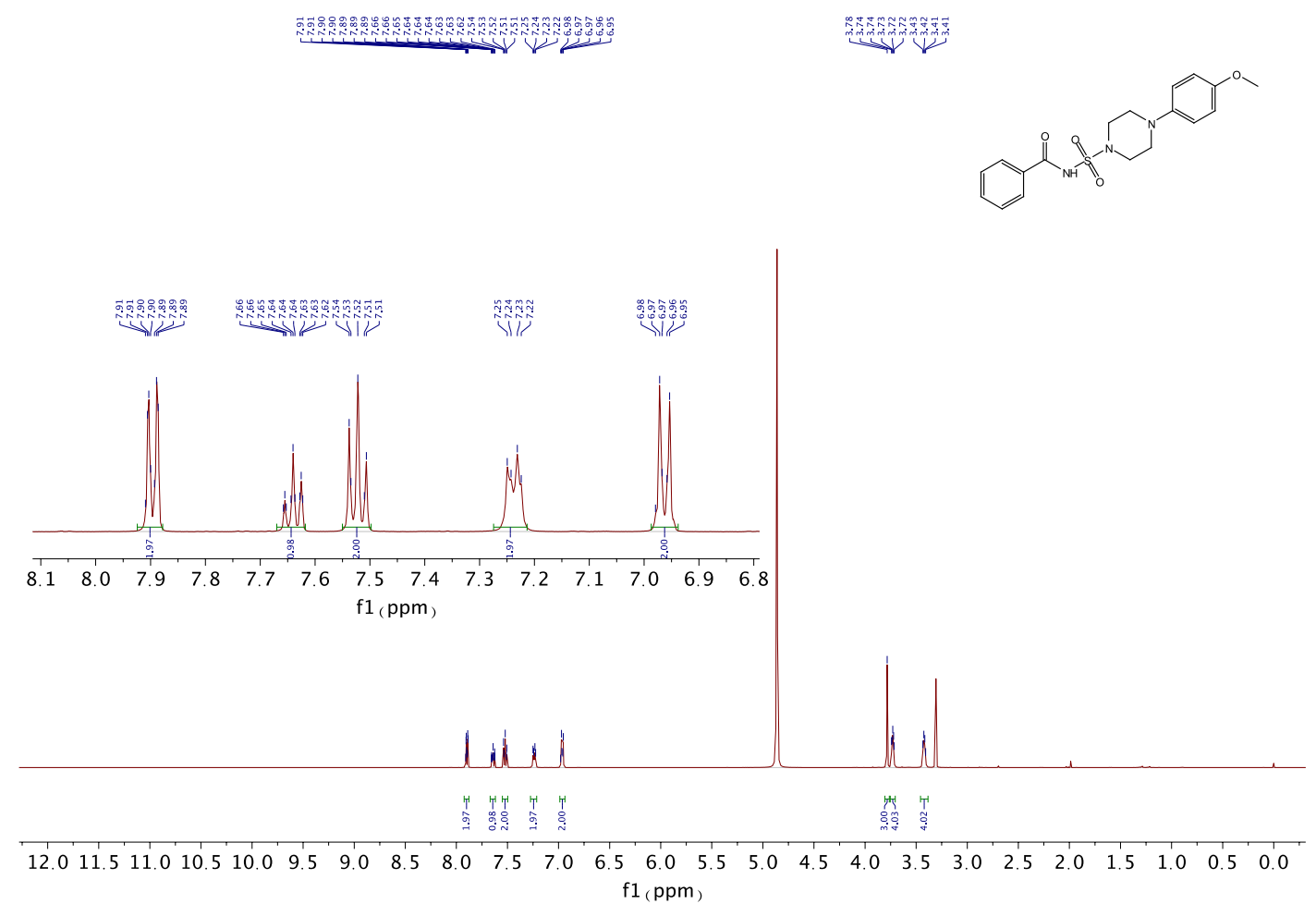

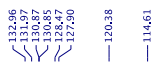

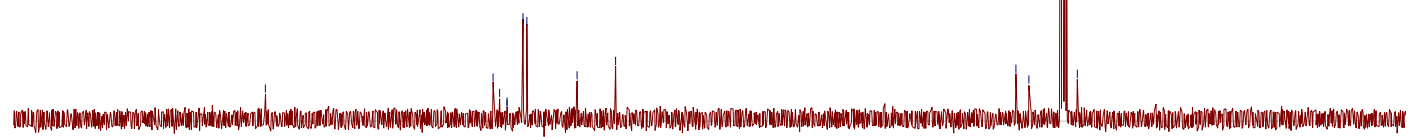

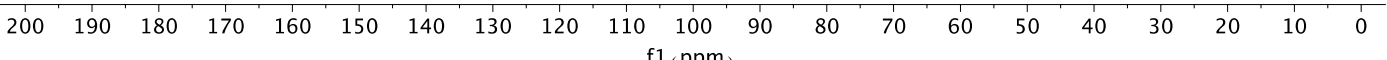



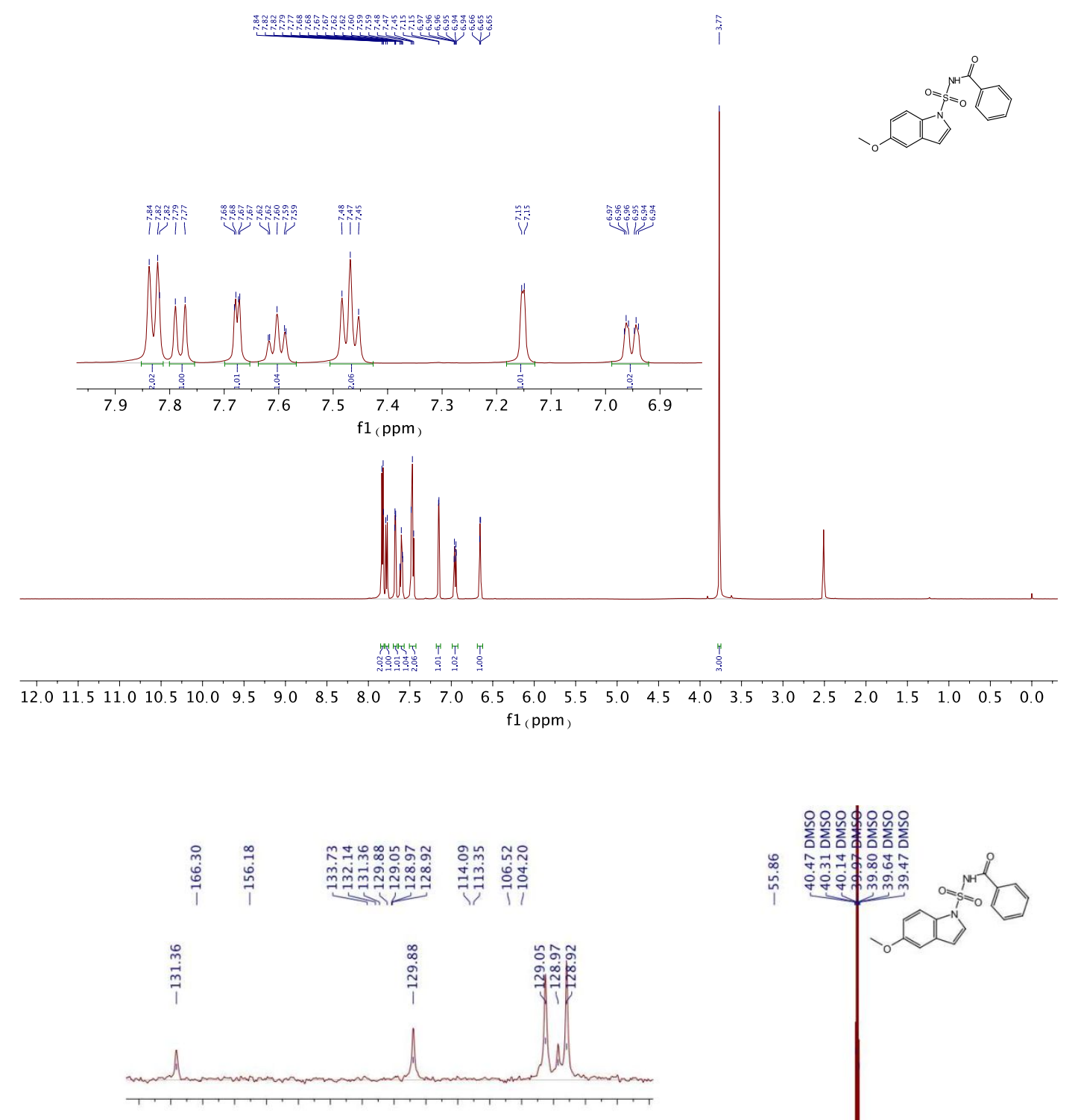

$\begin{array}{llllll}131.4 & 130.8 & 130.2 & 129.6 & 129.0 & 128.4\end{array}$ f1 ( $\mathrm{ppm}$ )

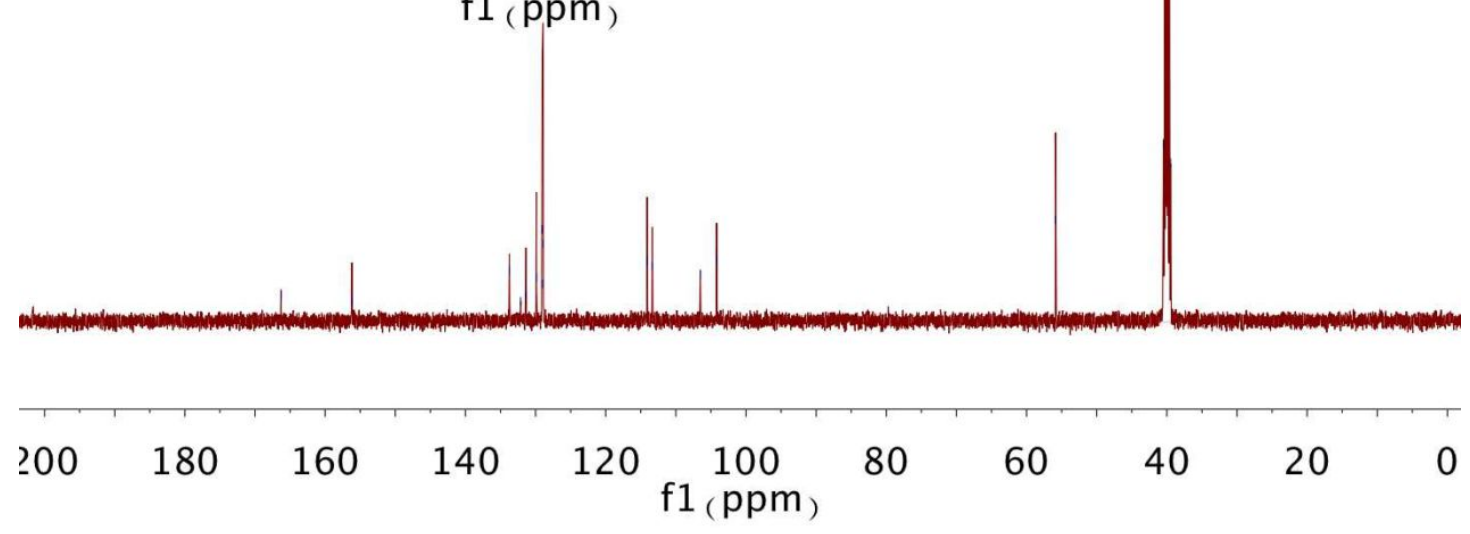




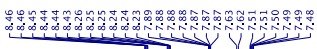
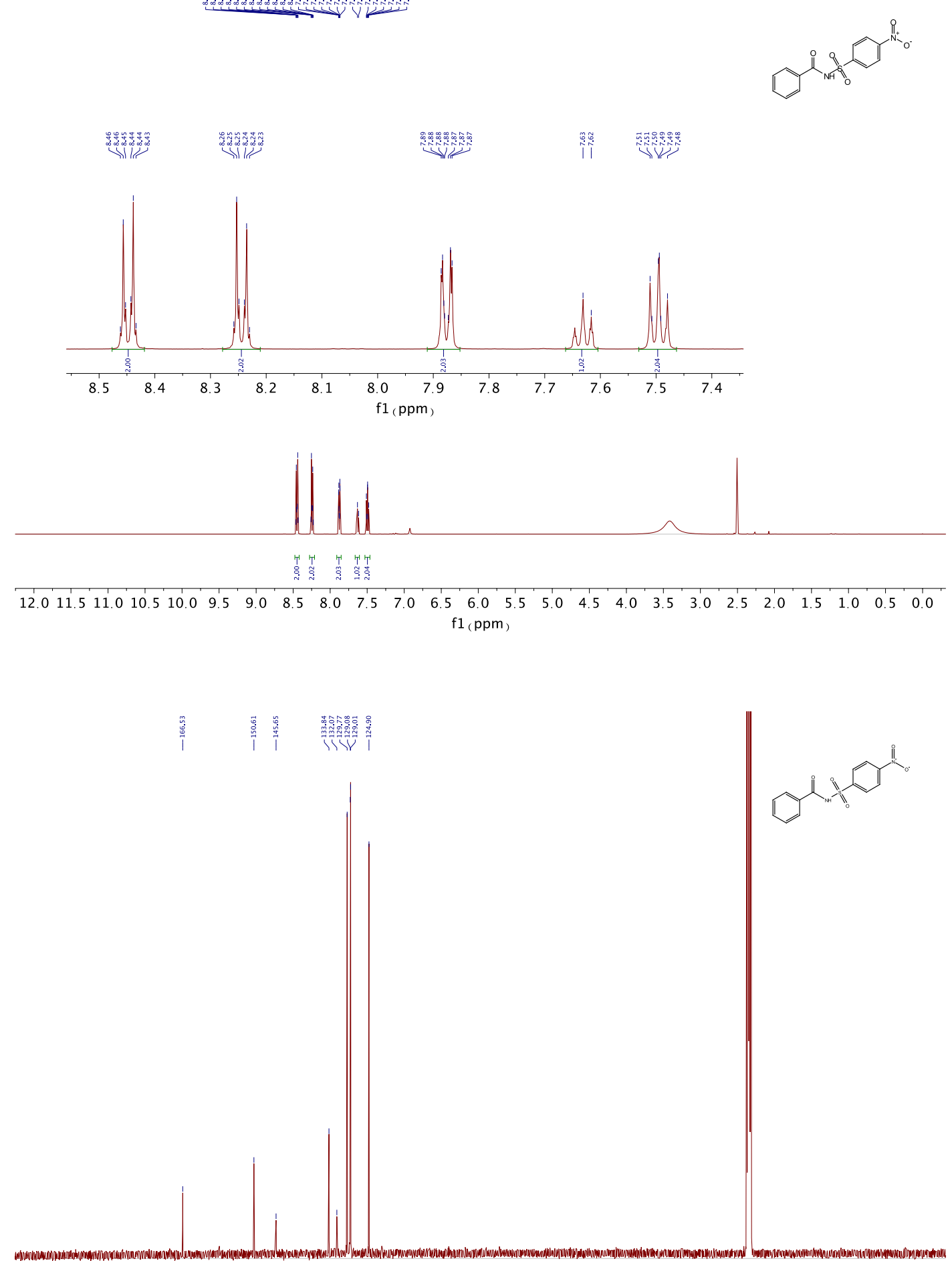

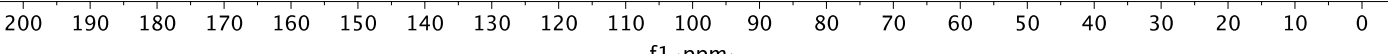


$N-((5-($ dimethylamino)naphthalen-1-yl)sulfonyl)benzamide (3p)
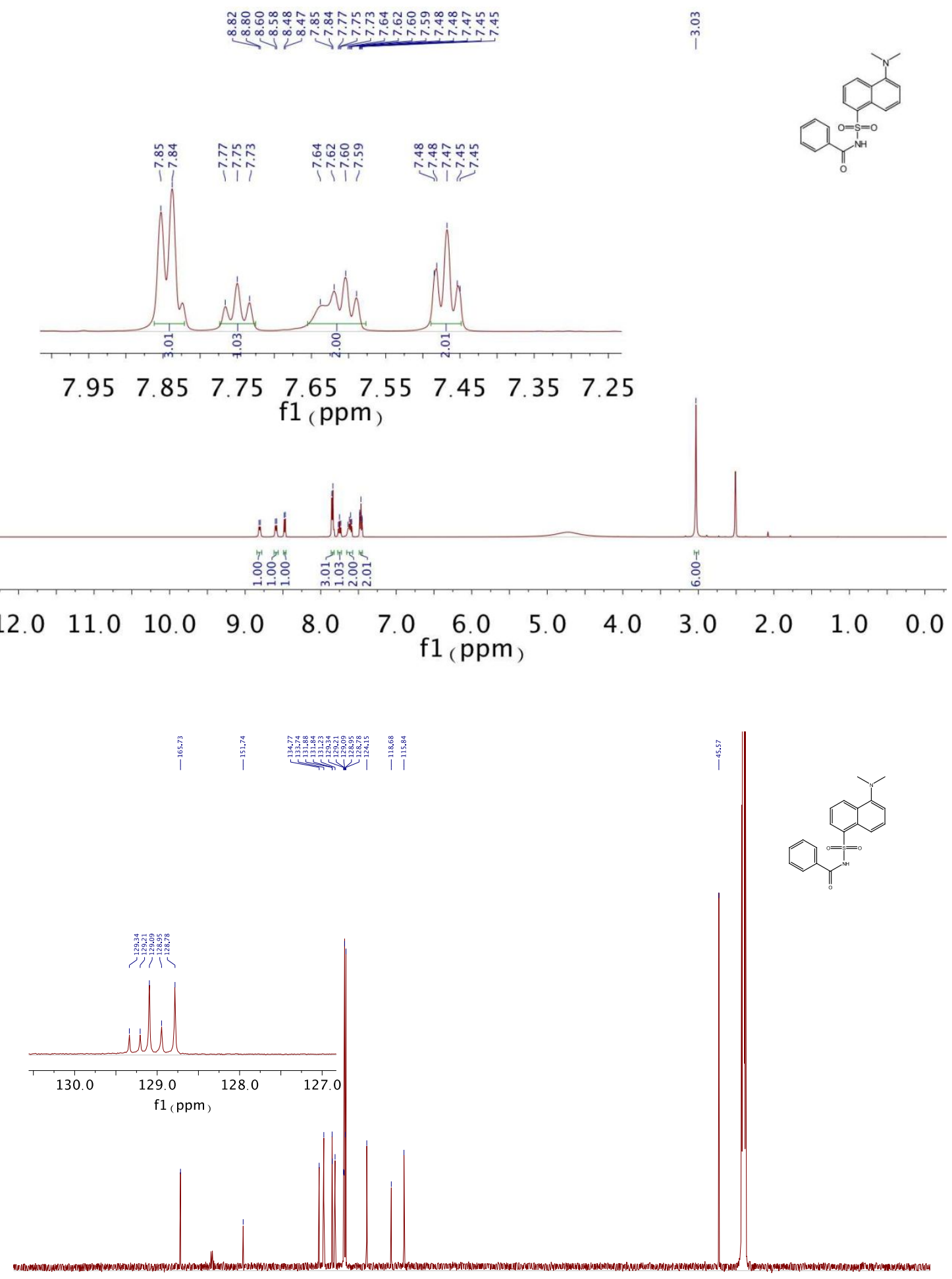

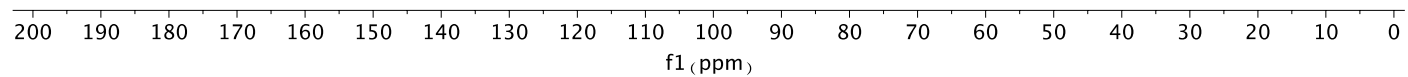


(8R,9S, 13S, 14S, 17S)-17-hydroxy-13-methyl-7,8,9,11,12,13,14,15,16,17-decahydro-6Hcyclopenta[a]phenanthren-3-yl benzoylsulfamate (3q)
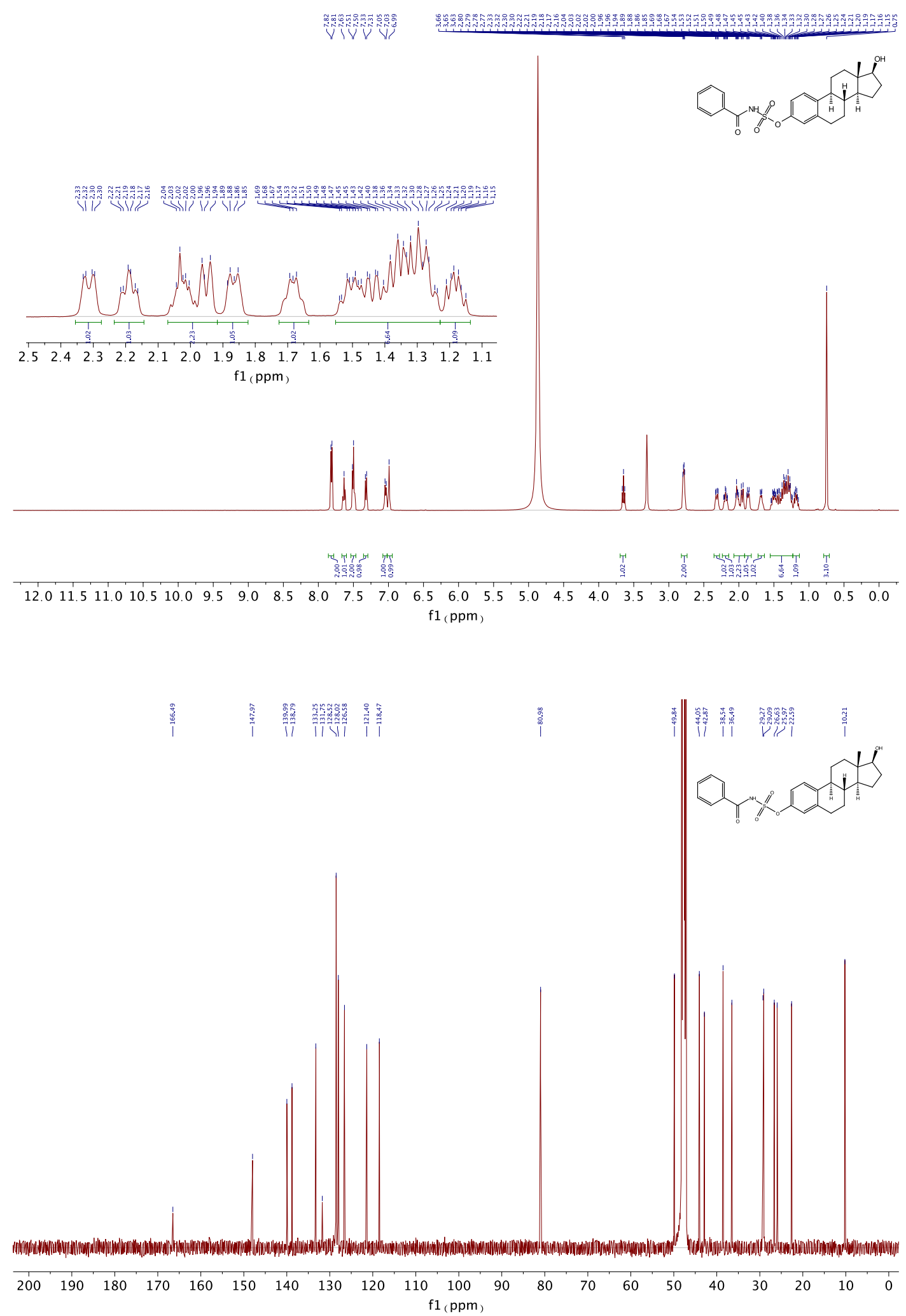
(7R,9S, 13S, 14S, 17S)-17-hydroxy-13-methyl-7-(9-((4,4,5,5,5-pentafluoropentyl)sulfinyl)nonyl)7,8,9,11,12,13,14,15,16,17-decahydro-6H-cyclopenta[a]phenanthren-3-yl benzoylsulfamate (3r)

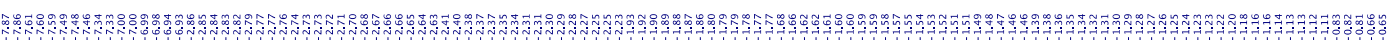
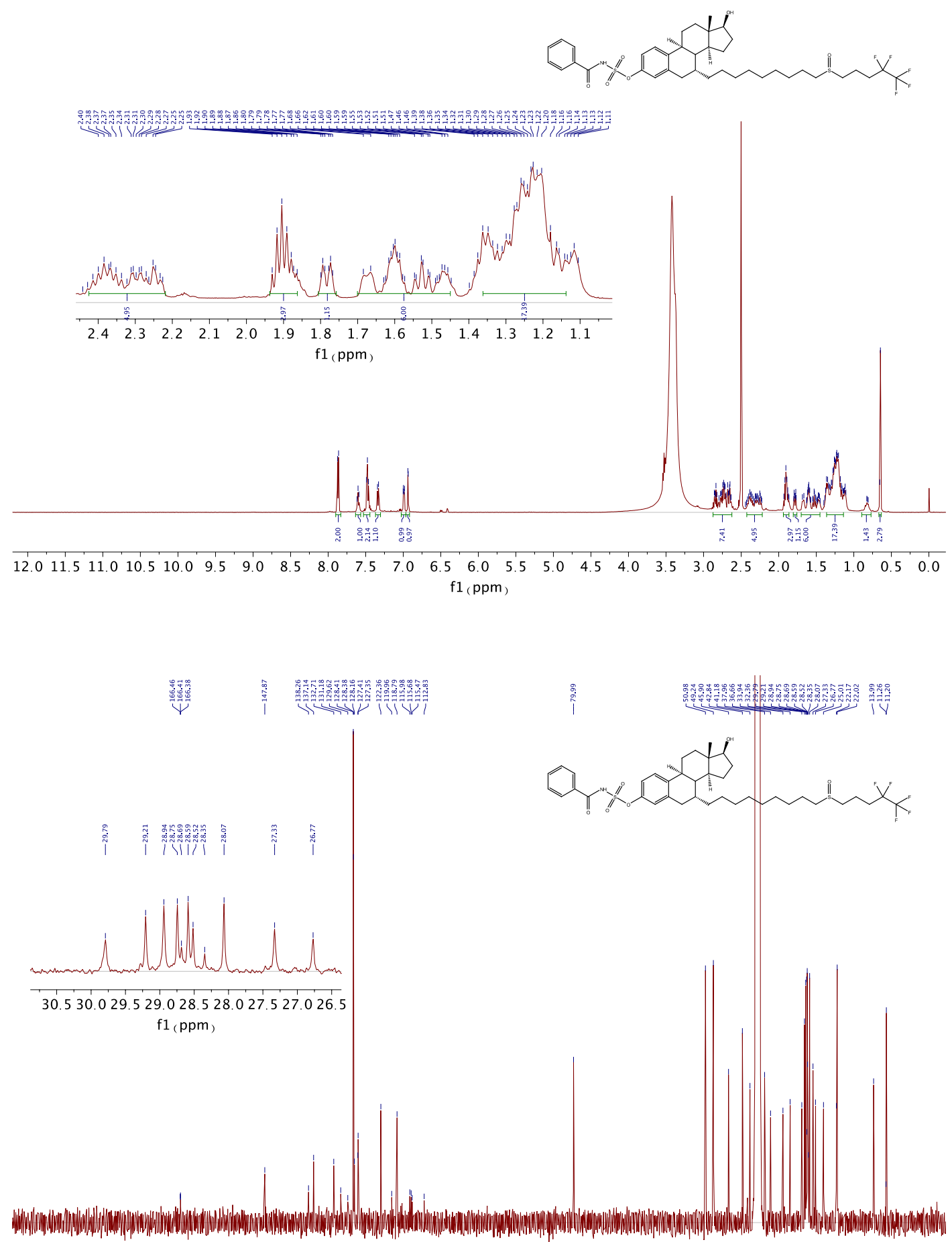

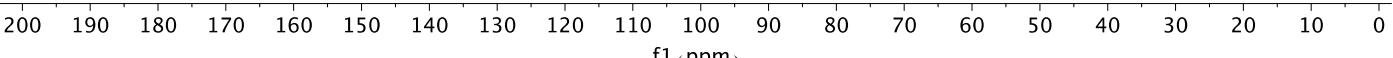


4-(((3R,4R)-4-(3,4-dimethoxybenzyl)-2-oxotetrahydrofuran-3-yl)methyl)-2-methoxyphenyl benzoylsulfamate (3s)

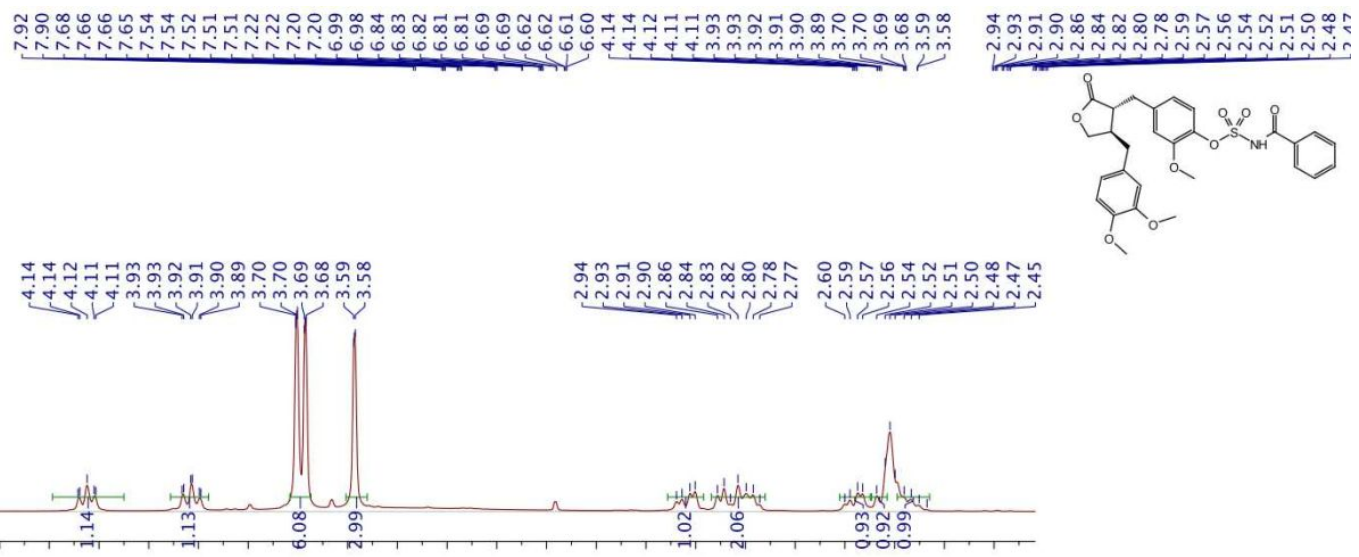

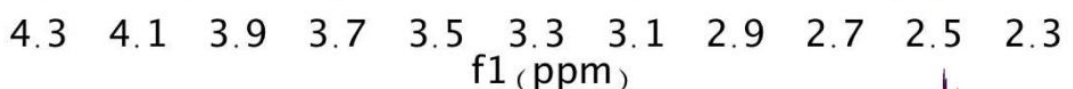
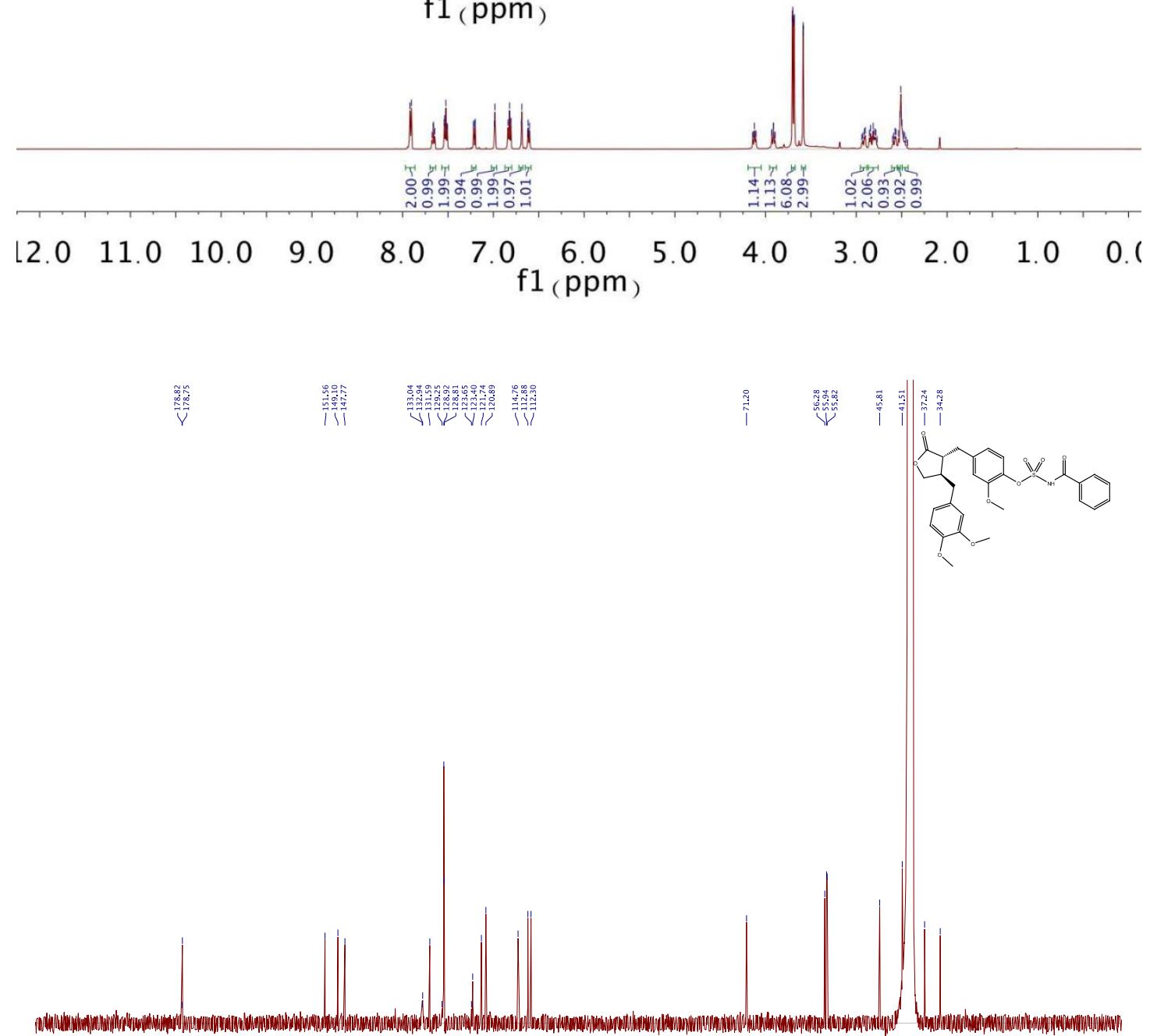

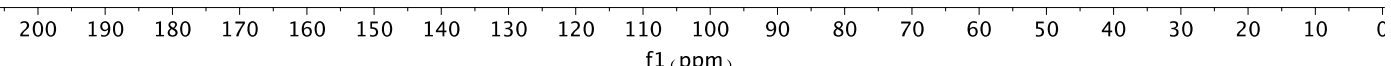

